\title{
MACLANE-VAQUIÉ CHAINS OF VALUATIONS ON A POLYNOMIAL RING
}

\author{
ENRIC NART
}

\begin{abstract}
Let $(K, v)$ be a valued field. We reinterpretate some results of MacLane and Vaquié on extensions of $v$ to valuations on the polynomial ring $K[x]$. We introduce certain MacLane-Vaquié chains constructed as a mixture of ordinary and limit augmentations of valuations. Every valuation $\nu$ on $K[x]$ is a limit (in a certain sense) of a countable MacLane-Vaquié chain. This chain underlying $\nu$ is essentially unique and contains discrete arithmetic data yielding an explicit description of the graded algebra of $\nu$ as an algebra over the graded algebra of $v$.
\end{abstract}

\section{INTRODUCTION}

Let $(K, v)$ be a valued field. In a pioneering work, S. MacLane studied the extensions of the valuation $v$ to the polynomial ring $K[x]$ in one indeterminate, in the case $v$ discrete of rank one [5, 6]. MacLane proved that all extensions of $v$ to $K[x]$ can be obtained as a limit of chains of augmented valuations:

$$
\mu_{0} \stackrel{\phi_{1}, \gamma_{1}}{\longrightarrow} \mu_{1} \stackrel{\phi_{2}, \gamma_{2}}{\longrightarrow} \cdots \longrightarrow \mu_{n-1} \stackrel{\phi_{n}, \gamma_{n}}{\longrightarrow} \mu_{n} \longrightarrow \cdots
$$

involving the choice of certain key polynomials $\phi_{n} \in K[x]$ and elements $\gamma_{n}$ belonging to some extension of the value group of $v$.

For valuations of arbitrary rank, different approaches to this problem were developed by V. Alexandru, N. Popescu and A. Zaharescu [1], by F.-V. Kuhlmann [4], and by F.J. Herrera, W. Mahboub, M.A. Olalla and M. Spivakovsky [2, 3].

This paper surveys the extension of MacLane's approach to the general case, developed by M. Vaquié [9, 10, 11]. In this general context, limit augmentations and the corresponding limit key polynomials appear as a new feature.

Starting with a valuation $\mu_{0}$ which admits key polynomials of degree one, we introduce MacLane-Vaquié chains as in (1), constructed as a mixture of ordinary and limit augmentations satisfying certain technical condition (Definition 4.1).

The main result, Theorem 4.3, states that all valuations $\nu$ on $K[x]$ extending $v$ fall in one, and only one, of the following cases:

(a) After a finite number $r$ of augmentation steps, we get $\mu_{r}=\nu$.

(b) After a finite number $r$ of augmentation steps, $\nu$ is the stable limit of some set of ordinary augmentations of $\mu_{r}$, defined by key polynomials of constant degree.

(c) It is the stable limit of a countably infinite chain of mixed augmentations as in (1), defined by key polynomials with unbounded degree.

2010 Mathematics Subject Classification. Primary 13A18; Secondary 12J20.

Key words and phrases. graded algebra, key polynomial, MacLane-Vaquié chain, valuation.

Partially supported by grant MTM2016-75980-P from the Spanish MEC. 
We say that $\nu$ has finite depth $r$, quasi-finite depth $r$, or infinite depth, respectively.

In section 5, we use this approach to obtain an explicit description of the structure of the graded algebra of $\nu$ as an algebra over the graded algebra of $v$, in terms of discrete arithmetic data supported by the underlying MacLane-Vaquié chain of $\nu$, which is essentially unique.

\section{KEY POLYNOMIALS OVER VALUED FIELDS}

Let $(K, v)$ be a non-trivially valued field. Let $k$ be the residue class field, $\Gamma=v\left(K^{*}\right)$ the value group and $\Gamma_{\mathbb{Q}}=\Gamma \otimes \mathbb{Q}$ the divisible hull of $\Gamma$.

Consider an extension of $v$ to the polynomial ring $K[x]$ in one indeterminate. That is, for some embedding $\Gamma \hookrightarrow \Lambda$ into another ordered abelian group, we consider a mapping whose restriction to $K$ is $v$,

$$
\mu: K[x] \longrightarrow \Lambda \infty
$$

and satisfies the following two conditions for all $f, g \in K[x]$ :

$$
\mu(f g)=\mu(f)+\mu(g), \quad \mu(f+g) \geq \operatorname{Min}\{\mu(f), \mu(g)\} .
$$

The support of $\mu$ is the prime ideal $\mathfrak{p}=\mathfrak{p}_{\mu}=\mu^{-1}(\infty) \in \operatorname{Spec}(K[x])$.

The value group of $\mu$ is the subgroup $\Gamma_{\mu} \subset \Lambda$ generated by $\mu(K[x] \backslash \mathfrak{p})$.

The valuation $\mu$ induces in a natural way a valuation $\bar{\mu}$ on the residue field $\kappa(\mathfrak{p})$, the field of fractions of $K[x] / \mathfrak{p}$. Let $k_{\mu}$ be the residue class field of $\bar{\mu}$.

Note that $\kappa(0)=K(x)$, while $\kappa(\mathfrak{p})$ is a simple finite extension of $K$ if $\mathfrak{p} \neq 0$.

The extension $\mu / v$ is commensurable if $\Gamma_{\mu} / \Gamma$ is a torsion group. In this case, there is a canonical embedding $\Gamma_{\mu} \hookrightarrow \Gamma_{\mathbb{Q}}$.

All valuations with non-trivial support are commensurable over $v$.

1.1. Graded algebra of a valuation. For any $\alpha \in \Gamma_{\mu}$, consider the abelian groups:

$$
\mathcal{P}_{\alpha}=\{g \in K[x] \mid \mu(g) \geq \alpha\} \supset \mathcal{P}_{\alpha}^{+}=\{g \in K[x] \mid \mu(g)>\alpha\} .
$$

The graded algebra of $\mu$ is the integral domain:

$$
\mathcal{G}_{\mu}:=\operatorname{gr}_{\mu}(K[x])=\bigoplus_{\alpha \in \Gamma_{\mu}} \mathcal{P}_{\alpha} / \mathcal{P}_{\alpha}^{+}
$$

There is a natural initial term mapping $\operatorname{in}_{\mu}: K[x] \rightarrow \mathcal{G}_{\mu}$, given by $\operatorname{in}_{\mu} \mathfrak{p}=0$ and

$$
\operatorname{in}_{\mu} g=g+\mathcal{P}_{\mu(g)}^{+} \in \mathcal{P}_{\mu(g)} / \mathcal{P}_{\mu(g)}^{+}, \quad \text { if } g \in K[x] \backslash \mathfrak{p} .
$$

There is a natural embedding of graded algebras $\mathcal{G}_{v}:=\operatorname{gr}_{v}(K) \hookrightarrow \mathcal{G}_{\mu}$.

If $\mu$ has non-trivial support $\mathfrak{p} \neq 0$, there is a natural isomorphism of graded algebras

$$
\mathcal{G}_{\mu} \simeq \operatorname{gr}_{\bar{\mu}}(\kappa(\mathfrak{p})) \text {. }
$$

The next definitions translate properties of the action of $\mu$ on $K[x]$ into algebraic relationships in the graded algebra $\mathcal{G}_{\mu}$.

Definition 1.1. Let $g, h \in K[x]$.

We say that $g, h$ are $\mu$-equivalent, and we write $g \sim_{\mu} h$, if $\operatorname{in}_{\mu} g=\operatorname{in}_{\mu} h$.

We say that $g$ is $\mu$-divisible by $h$, and we write $\left.h\right|_{\mu} g$, if $\operatorname{in}_{\mu} h \mid \operatorname{in}_{\mu} g$ in $\mathcal{G}_{\mu}$.

We say that $g$ is $\mu$-irreducible if $\left(\operatorname{in}_{\mu} g\right) \mathcal{G}_{\mu}$ is a non-zero prime ideal.

We say that $g$ is $\mu$-minimal if $g \nmid_{\mu} f$ for all non-zero $f \in K[x]$ with $\operatorname{deg}(f)<\operatorname{deg}(g)$.

The property of $\mu$-minimality admits a relevant characterization. 
Lemma 1.2. [8, Prop. 2.3] Let $\phi \in K[x]$ be a non-constant polynomial. Let

$$
f=\sum_{0 \leq s} a_{s} \phi^{s}, \quad a_{s} \in K[x], \quad \operatorname{deg}\left(a_{s}\right)<\operatorname{deg}(\phi)
$$

be the canonical $\phi$-expansion of $f \in K[x]$. Then, $\phi$ is $\mu$-minimal if and only if

$$
\mu(f)=\min \left\{\mu\left(a_{s} \phi^{s}\right) \mid 0 \leq s\right\}, \quad \forall f \in K[x] .
$$

1.2. Key polynomials. A (MacLane-Vaquié) key polynomial for $\mu$ is a monic polynomial in $K[x]$ which is simultaneously $\mu$-minimal and $\mu$-irreducible.

The set of key polynomials for $\mu$ is denoted $\operatorname{KP}(\mu)$.

All $\phi \in \mathrm{KP}(\mu)$ are irreducible in $K[x]$.

If $\mu$ has non-trivial support, the isomorphism of (2) shows that every non-zero homogeneous element of $\mathcal{G}_{\mu}$ is a unit. Thus, $\operatorname{KP}(\mu)=\emptyset$, because no polynomial in $K[x]$ can be $\mu$-irreducible.

If $\operatorname{KP}(\mu) \neq \emptyset$, the degree $\operatorname{deg}(\mu)$ is the minimal degree of a key polynomial for $\mu$.

Lemma 1.3. [8, Prop. 3.5] If $\operatorname{KP}(\mu) \neq \emptyset$, a non-zero homogeneous element $\operatorname{in}_{\mu} f$ is a unit in $\mathcal{G}_{\mu}$ if and only if $f \sim_{\mu} a$, for some $a \in K[x]$ with $\operatorname{deg}(a)<\operatorname{deg}(\mu)$. In this case, $\operatorname{in}_{\mu} f$ is algebraic over $\mathcal{G}_{v}$ and $\mu(f)$ belongs to $\Gamma_{\mathbb{Q}}$.

Theorem 1.4. [8, Thm. 3.9] Let $\phi \in \mathrm{KP}(\mu)$. For any monic $f \in K[x] \backslash K$, we have

$$
\mu(f) / \operatorname{deg}(f) \leq \mu(\phi) / \operatorname{deg}(\phi)
$$

and equality holds if and only if $f$ is $\mu$-minimal.

For any positive integer $m$, consider the subset

$$
\Gamma_{\mu, m}=\{\mu(a) \mid 0 \leq \operatorname{deg}(a)<m\} \subset \Gamma_{\mu} .
$$

For all $\chi \in \operatorname{KP}(\mu)$, the subset $\Gamma_{\mu, \operatorname{deg}(\chi)}$ is a subgroup of $\Gamma_{\mu}$ and $\left\langle\Gamma_{\mu, \operatorname{deg}(\chi)}, \mu(\chi)\right\rangle=\Gamma_{\mu}$.

Definition 1.5. If $\mathrm{KP}(\mu) \neq \emptyset$, the relative ramification index of $\mu$ is defined as:

$$
e:=e_{\mathrm{rel}}(\mu):=\left(\Gamma_{\mu}: \Gamma_{\mu, \operatorname{deg}(\mu)}\right) .
$$

By Lemma 1.3, $\Gamma_{\mu, \operatorname{deg}(\mu)} \subset \Gamma_{\mathbb{Q}}$. Hence, if $\mu / v$ is incommensurable, we have $e=\infty$.

If $\mu / v$ is commensurable, $e$ is the least positive integer such that $e \mu(\phi) \in \Gamma_{\mu, \operatorname{deg}(\mu)}$, where $\phi$ is any key polynomial for $\mu$ of minimal degree.

Definition 1.6. A key polynomial $\chi$ for $\mu$ is said to be proper if there exists some $\phi \in \mathrm{KP}(\mu)$ of minimal degree such that $\chi \chi_{\mu} \phi$.

For $\chi \in \mathrm{KP}(\mu)$, denote by $[\chi]_{\mu} \subset \mathrm{KP}(\mu)$ the subset of all key polynomials which are $\mu$-equivalent to $\chi$.

Since properness depends only on the class $[\chi]_{\mu}$, we may speak of proper and improper classes of $\mu$-equivalence of key polynomials for $\mu$.

If $e=1$, all classes are proper. If $e>1$, all key polynomials for $\mu$ of minimal degree are $\mu$-equivalent and form the unique improper class in $\mathrm{KP}(\mu) / \sim_{\mu}$ [8, Cor. 6.5].

Lemma 1.7. [8, Cor. 6.4] If $\chi$ is a proper key polynomial for $\mu$, then $\Gamma_{\mu, \operatorname{deg}(\chi)}=\Gamma_{\mu}$. 
Consider the subring of homogeneous elements of degree zero in the graded algebra

$$
\Delta=\Delta_{\mu}=\mathcal{P}_{0} / \mathcal{P}_{0}^{+} \subset \mathcal{G}_{\mu}
$$

There are canonical injective ring homomorphisms $k \hookrightarrow \Delta \hookrightarrow k_{\mu}$. We denote the algebraic closure of $k$ in $\Delta$ by

$$
\kappa=\kappa(\mu) \subset \Delta .
$$

This subfield satisfies $\kappa^{*}=\Delta^{*}$, where $\Delta^{*}$ is the multiplicative group of all units in $\Delta$.

The following results compute the ring $\Delta$ in terms of the presence (or absence) of key polynomials and the commensurability of $\mu$.

Theorem 1.8. [8, Thm. 4.4] The set $\mathrm{KP}(\mu)$ is empty if and only if all homogeneus elements in $\mathcal{G}_{\mu}$ are units. Equivalently, $\mu / v$ is commensurable and $\kappa=\Delta=k_{\mu}$ is an algebraic extension of $k$.

These valuations with $\operatorname{KP}(\mu)=\emptyset$ are said to be valuation-algebraic [4]. The valuations with $\operatorname{KP}(\mu) \neq \emptyset$ are valuation-transcendental. They split into two families.

Theorem 1.9. [8, Thm. 4.2] Suppose $\mu / v$ incommensurable. Let $\phi \in K[x]$ be a monic polynomial of minimal degree satisfying $\mu(\phi) \notin \Gamma_{\mathbb{Q}}$. Then, $\phi$ is a key polynomial for $\mu$, and $\mathrm{KP}(\mu)=[\phi]_{\mu}$. In particular, all key polynomials for $\mu$ are improper.

In this case, $\kappa=\Delta=k_{\mu}$ is a finite extension of $k$.

Theorem 1.10. [8, Thms. 4.5,4.6] Suppose $\mu / v$ commensurable and $\operatorname{KP}(\mu) \neq \emptyset$. Let $\phi$ be a key polynomial for $\mu$ of minimal degree $m$. Let $e=e_{\text {rel }}(\mu)$.

Let $u=\operatorname{in}_{\mu} a \in \mathcal{G}_{\mu}^{*}$, for some $a \in K[x]$ such that $\operatorname{deg}(a)<m$ and $\mu(a)=e \mu(\phi)$. Then, $\xi=\left(\operatorname{in}_{\mu} \phi\right)^{e} u^{-1} \in \Delta$ is transcendental over $k$ and satisfies $\Delta=\kappa[\xi]$.

Moreover, the canonical embedding $\Delta \hookrightarrow k_{\mu}$ induces an isomorphism $\kappa(\xi) \simeq k_{\mu}$.

These comensurable extensions $\mu / v$ admitting (MacLane-Vaquié) key polynomials are called residually transcendental valuations on $K[x]$.

The pair $\phi, u$ determines a residual polynomial operator

$$
R=R_{\mu, \phi, u}: K[x] \longrightarrow \kappa[y],
$$

which facilitates a complete description of the set $\operatorname{KP}(\mu)$, in terms of any fixed key polynomial of minimal degree.

Theorem 1.11. [8, Prop. 6.3] Suppose that $\mu$ is residually transcendental. Let $\phi \in \mathrm{KP}(\mu)$ of minimal degree $m$. A monic $\chi \in K[x]$ is a key polynomial for $\mu$ if and only if either

- $\operatorname{deg}(\chi)=m$ and $\chi \sim_{\mu} \phi$, or

- $\operatorname{deg}(\chi)=m e \operatorname{deg}(R(\chi))$ and $R(\chi)$ is irreducible in $\kappa[y]$.

Corollary 1.12. [8, Prop. 6.6] For any two key polynomials $\chi, \chi^{\prime} \in \operatorname{KP}(\mu)$, we have

$$
\left.\chi\right|_{\mu} \chi^{\prime} \Longleftrightarrow \chi \sim_{\mu} \chi^{\prime} \Longleftrightarrow R(\chi)=R\left(\chi^{\prime}\right) .
$$

In this case, $\operatorname{deg}(\chi)=\operatorname{deg}\left(\chi^{\prime}\right)$. 


\section{Chains of augmentations}

Let $\mu$ be a valuation on $K[x]$ extending the valuation $v$ on $K$.

Let $\Gamma_{\mu} \hookrightarrow \Lambda$ be an embedding of ordered groups, and let $\nu$ be a $\Lambda$-valued valuation whose restriction to $K$ is $v$. We say that $\mu \leq \nu$ if

$$
\mu(f) \leq \nu(f), \quad \forall f \in K[x]
$$

In this case, there is a canonical homomorphism of graded $\mathcal{G}_{v}$-algebras:

$$
\mathcal{G}_{\mu} \longrightarrow \mathcal{G}_{\nu}, \quad \operatorname{in}_{\mu} f \longmapsto \begin{cases}\operatorname{in}_{\nu} f, & \text { if } \mu(f)=\nu(f), \\ 0, & \text { if } \mu(f)<\nu(f) .\end{cases}
$$

A valuation $\mu$ is said to be maximal if it admits no strict upper bounds. In other words, $\mu \leq \nu$ implies $\mu=\nu$.

There are two perspectives concerning the comparison of $\mu$ with other valuations taking values in $\Lambda$. We may "look forward" and construct augmentations of $\mu$, or we may "look backward" and try to describe the set

$$
(-\infty, \mu)_{\Lambda}=\left\{\rho: K[x] \rightarrow \Lambda \infty \mid \rho \text { valuation, } \rho_{\mid K}=v, \rho<\mu\right\},
$$

usually with the purpose of finding "constructable" approximations to $\mu$ from below.

In the discrete-rank one case, MacLane designed concrete procedures in both directions [5], which were generalised by Vaquié to the general case [10, Sec. 1].

These procedures are summarized in Propositions 2.1 and 2.2 below.

2.1. Ordinary augmentations. Suppose $\operatorname{KP}(\mu) \neq \emptyset$. Choose $\phi \in \operatorname{KP}(\mu)$ and $\gamma \in$ $\Lambda \infty$ such that $\mu(\phi)<\gamma$.

The augmented valuation of $\mu$ with respect to this pair of data is the mapping

$$
\mu^{\prime}: K[x] \longrightarrow \Lambda \infty, \quad f=\sum_{0 \leq s} a_{s} \phi^{s} \longmapsto \mu^{\prime}(f)=\min \left\{\mu\left(a_{s}\right)+s \gamma \mid 0 \leq s\right\},
$$

defined in terms of $\phi$-expansions. We use the notation $\mu^{\prime}=[\mu ; \phi, \gamma]$. Note that $\mu^{\prime}(\phi)=\gamma$.

Proposition 2.1. [10, sec. 1.1], [8, sec. 7]

(1) The mapping $\mu^{\prime}=[\mu ; \phi, \gamma]$ is a valuation on $K[x]$.

If $\gamma<\infty$, it has trivial support. If $\gamma=\infty$, the support of $\mu^{\prime}$ is $\phi K[x]$.

(2) It satisfies $\mu<\mu^{\prime}$. Moreover, for a non-zero $f \in K[x]$, we have

$$
\mu(f)=\mu^{\prime}(f) \Longleftrightarrow \phi \nmid_{\mu} f .
$$

In this case, $\operatorname{in}_{\mu^{\prime}} f$ is a unit in $\mathcal{G}_{\mu^{\prime}}$.

(3) If $\gamma<\infty$, then $\phi$ is a key polynomial for $\mu^{\prime}$ of minimal degree. In particular, $\operatorname{deg}\left(\mu^{\prime}\right)=\operatorname{deg}(\phi)$.

(4) If $\phi$ is a proper key polynomial for $\mu$, then

$$
\Gamma_{\mu^{\prime}}=\left\langle\Gamma_{\mu}, \gamma\right\rangle, \quad \text { if } \gamma<\infty ; \quad \Gamma_{\mu^{\prime}}=\Gamma_{\mu}, \quad \text { if } \gamma=\infty .
$$

Proposition 2.2. [10, Thm. 1.15] If $\rho<\mu$, let $\Phi_{\rho, \mu}$ be the set of monic polynomials $\phi \in K[x]$ of minimal degree satisfying $\rho(\phi)<\mu(\phi)$.

Then, $\Phi_{\rho, \mu} \subset \operatorname{KP}(\rho)$ and for any $\phi \in \Phi_{\rho, \mu}$ we have

$$
\rho<[\rho ; \phi, \mu(\phi)] \leq \mu \text {. }
$$

For any non-zero $f \in K[x]$, the equality $\rho(f)=\mu(f)$ holds if and only if $\phi \nmid_{\rho} f$. 
The following observation is an immediate consequence of Propositions 2.1 and 2.2.

Theorem 2.3. A valuation $\mu$ is maximal if and only if $\operatorname{KP}(\mu)=\emptyset$.

Theorem 2.4 is another relevant consequence of Proposition 2.2. The proof given in [7, Thm. 3.9] for the group $\Lambda=\Gamma_{\mathbb{Q}}$ is valid for any ordered group $\Lambda$.

Theorem 2.4. For any valuation $\mu: K[x] \rightarrow \Lambda \infty$, the set $(-\infty, \mu)_{\Lambda}$ is totally ordered.

Let us derive some more practical consequences of Propositions 2.1 and 2.2.

Corollary 2.5. Take $\rho<\mu$ as above, and let $\nu$ be another valuation. Then,

(1) $\Phi_{\rho, \mu}=[\phi]_{\rho}$, for all $\phi \in \Phi_{\rho, \mu}$.

(2) $\rho<\mu<\nu \Longrightarrow \Phi_{\rho, \mu}=\Phi_{\rho, \nu}$. In particular,

$$
\rho(f)=\nu(f) \Longleftrightarrow \rho(f)=\mu(f), \quad \forall f \in K[x] .
$$

Proof. For all $\chi \in \mathrm{KP}(\rho), \phi \in \Phi_{\rho, \mu}$, Proposition 2.2 and Corollary 1.12 show that

$$
\left.\chi \in \Phi_{\rho, \mu} \Longleftrightarrow \phi\right|_{\rho} \chi \Longleftrightarrow \phi \sim_{\rho} \chi
$$

On the other hand, if $\rho<\mu<\nu$, let $\Phi_{\rho, \mu}=[\phi]_{\rho} \Phi_{\rho, \nu}=\left[\phi^{\prime}\right]_{\rho}$. Since

$$
\rho(\phi)<\mu(\phi) \leq \nu(\phi),
$$

Proposition 2.2 shows that $\left.\phi^{\prime}\right|_{\rho} \phi$. Thus, $\phi^{\prime} \sim_{\rho} \phi$, by Corollary 1.12 .

Corollary 2.6. Suppose that $\mu<\nu$ and let $\Phi_{\mu, \nu}=[\phi]_{\mu}$. Then,

(1) The kernel of the homomorphism $\mathcal{G}_{\mu} \rightarrow \mathcal{G}_{\nu}$ is the prime ideal $\left(\operatorname{in}_{\mu} \phi\right) \mathcal{G}_{\mu}$.

(2) All non-zero homogeneous elements in the image of $\mathcal{G}_{\mu} \rightarrow \mathcal{G}_{\nu}$ are units.

(3) If $\operatorname{KP}(\nu) \neq \emptyset$, then $\operatorname{deg}(\mu) \leq \operatorname{deg}(\nu)$.

Proof. Let $\rho=[\mu ; \phi, \nu(\phi)]$, and let in $\operatorname{in}_{\mu} f \in \mathcal{G}_{\mu}$ be a non-zero homogeneous element.

This element is mapped to zero in $\mathcal{G}_{\nu}$ if and only if $\mu(f)<\nu(f)$, and this is equivalent to $\left.\phi\right|_{\mu} f$ by Proposition 2.2. This proves (1).

By Proposition 2.2, $\mu<\rho \leq \nu$, and $\mathcal{G}_{\mu} \rightarrow \mathcal{G}_{\nu}$ is the composition of the canonical homomorphisms $\mathcal{G}_{\mu} \rightarrow \mathcal{G}_{\rho} \rightarrow \mathcal{G}_{\nu}$.

If $\operatorname{in}_{\mu} f$ is not mapped to zero in $\mathcal{G}_{\nu}$, then $\mu(f)=\rho(f)=\nu(f)$. Hence, the image of $\operatorname{in}_{\mu} f$ under the composition $\mathcal{G}_{\mu} \rightarrow \mathcal{G}_{\rho} \rightarrow \mathcal{G}_{\nu}$ is $\operatorname{in}_{\mu} f \mapsto \operatorname{in}_{\rho} f \mapsto \operatorname{in}_{\nu} f$.

By Proposition 2.1, in $f$ is a unit in $\mathcal{G}_{\rho}$. Thus, $\operatorname{in}_{\nu} f$ is a unit. This proves (2).

Since $\phi$ is a key polynomial for $\mu$, we have $\operatorname{deg}(\mu) \leq \operatorname{deg}(\phi)$.

On the other hand, all $a \in K[x]$ with $\operatorname{deg}(a)<\operatorname{deg}(\phi)$ satisfy $\mu(a)=\nu(a)$. Thus, $a$ cannot be a key polynomial for $\nu$. In fact, $\operatorname{in}_{\nu} a$ is a unit in $\mathcal{G}_{\nu}$ by item (2), because it is the image of $\operatorname{in}_{\mu} a$. Therefore, $\operatorname{deg}(\phi) \leq \operatorname{deg}(\nu)$ if $\operatorname{KP}(\nu) \neq \emptyset$. This proves (3).

Lemma 2.7. Let $\mu^{\prime}=[\mu ; \phi, \gamma]$ be an augmentation of $\mu$. For any $\delta \in \Lambda, \delta>\mu(\phi)$, consider the augmented valuation $\mu_{\delta}=[\mu ; \phi, \delta]$. Then,

$$
\left(\mu, \mu^{\prime}\right)_{\Lambda}:=\left\{\rho: K[x] \rightarrow \Lambda \infty \mid \rho \text { valuation, } \mu<\rho<\mu^{\prime}\right\}=\left\{\mu_{\delta} \mid \mu(\phi)<\delta<\gamma\right\} .
$$

Proof. For all $a \in K[x]$ of degree less than $\operatorname{deg}(\phi)$ we have $\mu(a)=\rho(a)=\mu^{\prime}(a)$ for all $\rho \in\left(\mu, \mu^{\prime}\right)_{\Lambda}$, by the definition of the augmented valuation.

If $\mu(\phi)<\delta<\gamma=\mu^{\prime}(\phi)$, then $\mu<\mu_{\delta}<\mu^{\prime}$, by their action on $\phi$-expansions.

Conversely, take any $\rho \in\left(\mu, \mu^{\prime}\right)_{\Lambda}$. By Corollary 2.5 (2), $\mu(\phi)<\rho(\phi)$. Also, we must have $\rho(\phi)<\gamma$ because otherwise we would have $\rho \geq \mu^{\prime}$, by their action on $\phi$-expansions. 
Since $\phi$ is a monic polynomial of minimal degree satisfying $\mu(\phi)<\rho(\phi)$, Proposition 2.2 shows that $\phi$ is a key polynomial for $\rho$. Hence, $\rho=\mu_{\delta}$, for $\delta=\rho(\phi)$, because both valuations coincide on $\phi$-expansions.

Unicity of ordinary augmentations. Let us analyze when different building data $\phi, \gamma$ yield the same ordinary augmentation of $\mu$.

Lemma 2.8. Let $\phi, \phi_{*} \in \operatorname{KP}(\mu)$. Let $\Gamma_{\mu} \hookrightarrow \Lambda$ be an embedding of ordered groups, and choose $\gamma, \gamma_{*} \in \Lambda \infty$ such that $\mu(\phi)<\gamma$ and $\mu\left(\phi_{*}\right)<\gamma_{*}$.

Then, $\mu^{\prime}=[\mu ; \phi, \gamma]$ coincides with $\mu_{*}^{\prime}=\left[\mu ; \phi_{*}, \gamma_{*}\right]$ if and only if

$$
\operatorname{deg}(\phi)=\operatorname{deg}\left(\phi_{*}\right) \quad \text { and } \quad \mu\left(\phi_{*}-\phi\right) \geq \gamma=\gamma_{*} .
$$

Proof. Suppose that the conditions of (3) hold. Let $\phi_{*}=\phi+a$ with $a \in K[x]$ of degree less than $\operatorname{deg}(\phi)$. By assumption, $\mu_{*}^{\prime}(a)=\mu^{\prime}(a)=\mu(a) \geq \gamma=\mu^{\prime}(\phi)$.

Hence, $\mu^{\prime}\left(\phi_{*}\right) \geq \gamma=\gamma_{*}=\mu_{*}^{\prime}\left(\phi_{*}\right)$. By comparison of their action on $\phi_{*}$-expansions we deduce that $\mu^{\prime} \geq \mu_{*}^{\prime}$. By the symmetry of (3), $\mu^{\prime}=\mu_{*}^{\prime}$.

Conversely, suppose $\mu^{\prime}=\mu_{*}^{\prime}$. By Proposition 2.1, $\phi, \phi_{*}$ are key polynomials for $\mu^{\prime}$ of minimal degree. Hence, $\operatorname{deg}(\phi)=\operatorname{deg}\left(\phi_{*}\right)$ and Theorem 1.4 shows that

$$
\gamma=\mu^{\prime}(\phi)=\mu^{\prime}\left(\phi_{*}\right)=\mu_{*}^{\prime}\left(\phi_{*}\right)=\gamma_{*} .
$$

In particular, $\mu\left(\phi_{*}-\phi\right)=\mu^{\prime}\left(\phi_{*}-\phi\right) \geq \gamma$.

2.2. Depth zero valuations. Take $a \in K, \gamma \in \Lambda \infty$. The following valuation $\mu=\omega_{a, \gamma}$ on $K[x]$ is said to be a depth zero valuation:

$$
\mu\left(\sum_{0 \leq s} a_{s}(x-a)^{s}\right)=\min \left\{v\left(a_{s}\right)+s \gamma \mid 0 \leq s\right\} .
$$

If $\gamma<\infty$, then $x-a$ is a key polynomial for $\mu$; hence, $\operatorname{deg}(\mu)=1$.

It is easy to check that

$$
\begin{gathered}
\omega_{a, \gamma} \leq \omega_{b, \delta} \Longleftrightarrow \gamma \leq \delta \quad \text { and } \quad v(a-b) \geq \gamma \\
\left(-\infty, \omega_{a, \gamma}\right)_{\Lambda}=\left\{\omega_{a, \delta} \mid \delta \in \Lambda, \delta<\gamma\right\} .
\end{gathered}
$$

2.3. Degree of maximal valuations. Let $\mu$ be a valuation on $K[x]$, and let $\Gamma_{\mu} \hookrightarrow \Lambda$ be an embedding of $\Gamma_{\mu}$ into a dense ordered group. That is, for any $\beta<\gamma$ in $\Lambda$, there exists $\delta \in \Lambda$ such that $\beta<\delta<\gamma$. For instance, $\Lambda=\left(\Gamma_{\mu}\right)_{\mathbb{Q}}$ satisfies this condition.

By Proposition 2.2, any valuation $\rho<\mu$ admits key polynomials and $\operatorname{deg}(\rho)$ is defined. For any open interval $I$ of valuations we may consider its set of degrees:

$$
\operatorname{Deg}(I)=\{\operatorname{deg}(\rho) \mid \rho \in I\} \subset \mathbb{N} .
$$

For instance, if $\mu_{0}=\omega_{a, \gamma}$ is a depth zero valuation, we deduce from (4) that

$$
\operatorname{Deg}\left(\left(-\infty, \mu_{0}\right)_{\Lambda}\right)=\{1\} \text {. }
$$

Also, for an augmented valuation $\mu^{\prime}=[\mu ; \phi, \gamma]$, Lemma 2.7 shows that

$$
\operatorname{Deg}\left(\left(\mu, \mu^{\prime}\right)_{\Lambda}\right)=\{\operatorname{deg}(\phi)\}
$$

If $\mu$ is a maximal valuation, we may define

$$
\operatorname{deg}(\mu):=\sup \left(\operatorname{Deg}\left((-\infty, \mu)_{\left(\Gamma_{\mu}\right)_{\mathbb{Q}}}\right)\right) \in \mathbb{N} \infty
$$

We shall see in section 4 that there are maximal valuations of infinite degree. 


\section{Limit AUGMENTATiONS}

Consider two valuations $\mu, \nu$ with values in a common ordered group, such that $\mu<\nu$. An iterative application of Proposition 2.2. yields a chain of ordinary augmentations

$$
\mu<\mu_{1}<\cdots<\mu_{n}<\cdots \leq \nu
$$

each one getting closer to $\nu$ than the previous one. Unfortunately, this chain does not always "converge" to $\nu$.

In order to overcome this obstacle, Vaquié introduced limit augmentations based on certain limit key polynomials.

This section is devoted to discuss these limit augmentations.

3.1. Continuous families of augmentations. Let $A$ be a totally ordered set.

A totally ordered family of valuations parameterized by $A$ is a totally ordered set

$$
\mathcal{A}=\left(\rho_{i}\right)_{i \in A}
$$

of valuations taking values in a common ordered group, such that the bijection $i \rightarrow \rho_{i}$ is an isomorphism of ordered sets between $A$ and $\mathcal{A}$.

A polynomial $f \in K[x]$ is $\mathcal{A}$-stable if for some index $i_{0} \in A$ we have

$$
\rho_{i}(f)=\rho_{i_{0}}(f), \quad \forall i \geq i_{0} .
$$

This stable value is denoted $\rho_{\mathcal{A}}(f)$.

We obtain in this way a stability function $\rho_{\mathcal{A}}$ which is defined only on the multiplicatively closed subset of $K[x]$ formed by the stable polynomials.

By Corollary 2.5 (2), the instability of $f \in K[x]$ is characterised as follows:

$$
f \text { unstable } \Longleftrightarrow \rho_{i}(f)<\rho_{j}(f), \quad \forall i<j .
$$

We say that $\mathcal{A}$ has a stable limit if all polynomials in $K[x]$ are stable. In this case, $\rho_{\mathcal{A}}$ is a valuation on $K[x]$, which is called the stable limit of $\mathcal{A}$. We write $\rho_{\mathcal{A}}=\lim _{i \in A} \rho_{i}$.

Proposition 3.1. Let $\mathcal{A}=\left(\rho_{i}\right)_{i \in A}$ be a totally ordered family of valuations having a stable limit. If the set $A$ contains no maximal element, the valuation $\rho_{\mathcal{A}}$ has trivial support and satisfies $\operatorname{KP}\left(\rho_{\mathcal{A}}\right)=\emptyset$. In particular, $\rho_{\mathcal{A}}$ is a maximal valuation.

Proof. Since $A$ has no maximal element, we have $\rho_{i}<\rho_{\mathcal{A}}$ for all $i \in A$. By Theorem 2.3, all the valuations $\rho_{i}$ have trivial support. All non-zero $f \in K[x]$ satisfy $\rho_{\mathcal{A}}(f)=\rho_{i}(f)<\infty$ for some $i \in A$. Therefore, $\rho_{\mathcal{A}}$ has trivial support too.

All non-zero homogeneous elements in $\mathcal{G}_{\rho_{\mathcal{A}}}$ are units by Corollary 2.6. By Theorem 1.8. $\operatorname{KP}\left(\rho_{\mathcal{A}}\right)=\emptyset$ and $\rho_{\mathcal{A}}$ is maximal by Theorem 2.3 .

Let us focus our attention on totally ordered families of valuations of a special type.

Definition 3.2. Let $\mu$ be a non-maximal valuation and let $\Gamma_{\mu} \hookrightarrow \Lambda$ be an embedding of ordered groups. Consider a family of ordinary augmentations of $\mu$,

$$
\mathcal{A}=\left(\rho_{i}\right)_{i \in A}, \quad \rho_{i}=\left[\mu ; \chi_{i}, \beta_{i}\right], \quad \beta_{i} \in \Lambda, \beta_{i}>\mu\left(\chi_{i}\right),
$$

parameterized by a totally ordered set $A$ such that $\beta_{i}<\beta_{j}$ for all $i<j$ in $A$.

$W e$ say that $\mathcal{A}$ is a continuous family of augmentations of $\mu$ of stable degree $m$ it it satisfies the following conditions: 
(1) The set $A$ contains no maximal element.

(2) All key polynomials $\chi_{i} \in \mathrm{KP}(\mu)$ have degree $m$.

(3) For all $i<j$ in $A, \chi_{j}$ is a key polynomial for $\rho_{i}$ and satisfies

$$
\chi_{j} \chi_{\rho_{i}} \chi_{i} \text { and } \rho_{j}=\left[\rho_{i} ; \chi_{j}, \beta_{j}\right] .
$$

The basic examples of continuous families of augmentations are provided by certain valuations $\nu$ on $K[x]$ such that $\mu<\nu$.

We denote the common degree of all polynomials in the set $\Phi_{\mu, \nu}$ by $\operatorname{deg}\left(\Phi_{\mu, \nu}\right)$.

Proposition 3.3. Let $\nu$ be a valuation on $K[x]$ such that $\mu<\nu$. Suppose that the set $A=\nu\left(\Phi_{\mu, \nu}\right)$ does not contain a maximal element in $\left(\Gamma_{\nu}\right) \infty$. For all $\alpha \in A$, choose some polynomial $\chi_{\alpha} \in \Phi_{\mu, \nu}$ such that $\nu\left(\chi_{\alpha}\right)=\alpha$, and build $\rho_{\alpha}=\left[\mu ; \chi_{\alpha}, \alpha\right]$. Then, $\mathcal{A}=\left(\rho_{\alpha}\right)_{\alpha \in A}$ is a continuous family of augmentations of $\mu$ of stable degree $m=\operatorname{deg}\left(\Phi_{\mu, \nu}\right)$.

Moreover, all polynomials in $K[x]$ of degree $m$ are stable.

Proof. Clearly, the family $\mathcal{A}$ satisfies conditions (1) and (2) of Definition 3.2

For all $a \in K[x]$ with $\operatorname{deg}(a)<m$, we have $\mu(a)=\nu(a)$ by the definition of $\Phi_{\mu, \nu}$, and $\mu(a)=\rho_{\alpha}(a)$ for all $\alpha \in A$, by the definition of the augmented valuation $\rho_{\alpha}$.

For $\alpha<\beta$ in $A$, write $\chi_{\beta}=\chi_{\alpha}+a$ for some $a \in K[x]$ of degree less than $m$. Since $\nu\left(\chi_{\alpha}\right)=\alpha<\beta=\nu\left(\chi_{\beta}\right)$, we deduce that $\mu(a)=\nu(a)=\alpha$. By the definition of the augmented valuations,

$$
\rho_{\alpha}\left(\chi_{\beta}\right)=\alpha<\beta=\rho_{\beta}\left(\chi_{\beta}\right), \quad \rho_{\alpha}\left(\chi_{\alpha}\right)=\alpha=\rho_{\beta}\left(\chi_{\alpha}\right) .
$$

By Corollary 2.5, $\chi_{\alpha} \notin \Phi_{\rho_{\alpha}, \rho_{\beta}}=\left[\chi_{\beta}\right]_{\rho_{\alpha}}$. Thus, $\chi_{\beta}$ is a key polynomial for $\rho_{\alpha}$ and $\chi_{\alpha} \chi_{\rho_{\alpha}} \chi_{\beta}$

Also, $\left[\rho_{\alpha} ; \chi_{\beta}, \beta\right]=\rho_{\beta}$, because both valuations coincide on $\chi_{\beta}$-expansions. This proves that $\mathcal{A}$ satisfies (3).

Finally, let us see that all monic $f \in K[x]$ of degree $m$ are stable. Suppose that $f$ is unstable, and write $f=\chi_{\beta}+a_{\beta}$ for all $\beta \in A$, where $a_{\beta} \in K[x]$ has degree less than $m$. Then, for all $\alpha<\beta \in A$, we have

$$
\mu(f) \leq \rho_{\alpha}(f)<\rho_{\beta}(f)=\min \left\{\beta, \nu\left(a_{\beta}\right)\right\} \leq \nu(f) .
$$

This implies $f \in \Phi_{\rho_{\alpha}, \nu} \subset \Phi_{\mu, \nu}$; in particular, $\nu(f) \in A$. On the other hand, $\Phi_{\rho_{\alpha}, \nu}=$ $\left[\chi_{\beta}\right]_{\rho_{\alpha}}$, so that $f \sim_{\rho_{\alpha}} \chi_{\beta}$ and $\rho_{\alpha}(f)=\rho_{\alpha}\left(\chi_{\beta}\right)=\alpha$. We deduce from (6) that $\alpha<\nu(f)$ for all $\alpha \in A$. This is impossible, because $\nu(f) \in A$.

The following properties hold for all continuous families of augmentations, and they are easily deduced from the definitions.

- $\mathcal{A}$ is a totally ordered family of valuations, parameterized by the set $A$.

- All polynomials in $K[x]$ of degree less than $m$ are stable.

- For all $i, j \in A, \rho_{i}\left(\chi_{j}\right)=\min \left\{\beta_{i}, \beta_{j}\right\}$.

- For all $i \in A, \operatorname{deg}\left(\rho_{i}\right)=m$ and $\chi_{i}$ is a proper key polynomial for $\rho_{i}$. By Theorem 1.9, $\rho_{i}$ is residually transcendental.

- For all $i<j$ in A, $\Phi_{\rho_{i}, \rho_{j}}=\left[\chi_{j}\right]_{\rho_{i}}$ and this class is proper.

By the definition of the augmented valuation, $\Gamma_{\rho_{i}, m}=\Gamma_{\rho_{j}, m}$ for all $i<j$. Thus, Lemma 1.7 shows that

$$
\Gamma_{\rho_{i}}=\Gamma_{\rho_{i}, m}=\Gamma_{\rho_{j}, m}=\Gamma_{\rho_{j}} .
$$


In particular, $\rho_{i}$ has relative ramification index equal to one.

The common value grup $\Gamma_{\mathcal{A}}:=\Gamma_{\rho_{i}}$ for all $i \in A$, is called the stable value group of the continuous family. Note that $\beta_{i} \in \Gamma_{\mathcal{A}}$ for all $i$.

The class $\Phi_{\mu, \mathcal{A}}:=\Phi_{\mu, \rho_{i}}=\left[\chi_{i}\right]_{\mu}$ does not depend on $i$, by Corollary 2.5 (2). The following result is a consequence of Lemma 1.7, by the same argument as in (7).

Lemma 3.4. If the class $\Phi_{\mu, \mathcal{A}}$ is proper, then $\Gamma_{\mu}=\Gamma_{\mathcal{A}}$.

Remark. Any cofinal family of $\left(\rho_{i}\right)_{i \in A}$ will have the same limit behaviour. Since all totally ordered sets admit well-ordered cofinal subsets, we may always assume that the set $A$ is well-ordered.

Essential continuous families of augmentations. Let $m_{\infty}$ be the minimal degree of an unstable polynomial. We agree that $m_{\infty}=\infty$ if all polynomials are stable.

Any continuous family falls in one of the following three cases:

(a) It has a stable limit. That is, $m_{\infty}=\infty$, so that the function $\rho_{\mathcal{A}}$ is a valuation on $K[x]$. This valuation is commensurable and satisfies $\operatorname{KP}\left(\rho_{\mathcal{A}}\right)=\emptyset$.

(b) It is inessential. That is, $m_{\infty}=m$.

(c) It is essential. That is, $m<m_{\infty}<\infty$.

Let $\nu$ be a valuation on $K[x]$ such that $\rho_{i}<\nu$ for all $i \in A$.

If $\left(\rho_{i}\right)_{i \in A}$ is inessential and $f \in K[x]$ is an unstable polynomial of degree $m$, then the ordinary augmentation $\mu^{\prime}=[\mu ; f, \nu(f)]$ satisfies

$$
\rho_{i}<\mu^{\prime} \leq \nu, \quad \forall i \in A .
$$

In other words, $\mu^{\prime}$ is closer to $\nu$ than any $\rho_{i}$, and $\mu^{\prime}$ is obtained from $\mu$ by a single augmentation. This justifies why we call it "inessential".

3.2. Limit key polynomials. Let us fix an essential continuous family $\mathcal{A}=\left(\rho_{i}\right)_{i \in A}$ of augmentations of $\mu$ of stable degree $m$.

We define the set $\mathrm{KP}_{\infty}(\mathcal{A})$ of limit key polynomials for $\mathcal{A}$ as the set of monic unstable polynomials in $K[x]$ of minimal degree $m_{\infty}$. We recall that $m_{\infty}>m$.

Let us fix a limit key polynomial $\phi \in \operatorname{KP}_{\infty}(\mathcal{A})$. Since the product of stable polynomials is stable, $\phi$ is irreducible in $K[x]$.

Let $\Gamma_{\mathcal{A}} \hookrightarrow \Lambda$ be an embedding of ordered groups, and choose $\gamma \in \Lambda \infty$ such that $\rho_{i}(\phi)<\gamma$ for all $i \in A$. We denote by $\mu^{\prime}=[\mathcal{A} ; \phi, \gamma]$ the following mapping:

$$
\mu^{\prime}: K[x] \longrightarrow \Lambda \infty, \quad f=\sum_{0 \leq s} a_{s} \phi^{s} \longmapsto \mu^{\prime}(f)=\min \left\{\rho_{\mathcal{A}}\left(a_{s}\right)+s \gamma \mid 0 \leq s\right\},
$$

defined in terms of $\phi$-expansions. Note that $\mu^{\prime}(\phi)=\gamma$.

This limit augmentation shares some features with the ordinary augmentations.

Proposition 3.5. [10, Sec. 1.4], [8, Sec. 7]

(1) The mapping $\mu^{\prime}=[\mathcal{A} ; \phi, \gamma]$ is a valuation on $K[x]$.

If $\gamma<\infty$, it has trivial support. If $\gamma=\infty$, the support of $\mu^{\prime}$ is $\phi K[x]$.

(2) For all $i \in A$ we have $\rho_{i}<\mu^{\prime}$. Moreover, for all non-zero $f \in K[x]$,

$$
f \text { is stable } \Longleftrightarrow \exists i \in A \text { such that } \rho_{i}(f)=\mu^{\prime}(f) \text {. }
$$

In this case, $\operatorname{in}_{\mu^{\prime}} f$ is a unit in $\mathcal{G}_{\mu^{\prime}}$. 
(3) If $\gamma<\infty$, then $\phi$ is a key polynomial for $\mu^{\prime}$, of minimal degree. In particular, $\operatorname{deg}\left(\mu^{\prime}\right)=\operatorname{deg}(\phi)=m_{\infty}$.

(4) Suppose that the class $\Phi_{\mu, \mathcal{A}}$ is proper. Then,

$$
\Gamma_{\mu^{\prime}}=\left\langle\Gamma_{\mu}, \gamma\right\rangle, \quad \text { if } \gamma<\infty ; \quad \Gamma_{\mu^{\prime}}=\Gamma_{\mu}, \quad \text { if } \gamma=\infty .
$$

Example. Let $p$ be a prime number and consider the $p$-adic valuation $v=\operatorname{ord}_{p}$ on $\mathbb{Q}$. Take a $p$-adic number with non-zero $p$-adic coefficients

$$
\theta=\sum_{i=0}^{\infty} c_{i} p^{i} \in \mathbb{Z}_{p}, \quad c_{i} \in\{1, \ldots, p-1\},
$$

and denote its partial sums by $a_{i}=\sum_{0 \leq j<i} c_{i} p^{i} \in \mathbb{Z}$, for all $i>0$.

Consider the depth zero valuation $\mu=\omega_{0,0}$ and the chain of ordinary augmentations

$$
\mu \stackrel{x-a_{1}, 1}{\longrightarrow} \rho_{1} \stackrel{x-a_{2}, 2}{\longrightarrow} \cdots \longrightarrow \rho_{i-1} \stackrel{x-a_{i}, i}{\longrightarrow} \rho_{i} \longrightarrow \cdots
$$

All these valuations have depth zero and are augmentations of $\mu$ :

$$
\rho_{i}=\omega_{a_{i}, i}=\left[\mu ; x-a_{i}, i\right] .
$$

They form a continuous family $\mathcal{A}=\left(\rho_{i}\right)_{i \geq 1}$ of augmentations of $\mu$. One checks easily that a non-zero $f \in \mathbb{Q}[x]$ is stable if and only if $f(\theta) \neq 0$.

Let us analyze the different possibilities for the limit of this family.

- If $\theta \in \mathbb{Q}$, then $x-\theta$ is an unstable polynomial of degree one. Thus, the family is inessential. The natural limit valuation $\mu^{\prime}=\omega_{\theta, \infty}$ has depth zero.

- If $\theta$ is transcendental over $\mathbb{Q}$, then the family $\mathcal{A}$ has a stable limit $\mu^{\prime}=\rho_{\mathcal{A}}$.

With the notation of the introduction, $\mu^{\prime}$ has quasi-finite depth zero.

- If $\theta \in \overline{\mathbb{Q}} \backslash \mathbb{Q}$, the family is essential and the minimal polynomial $\phi$ of $\theta$ over $\mathbb{Q}$ is a limit key polynomial for $\mathcal{A}$. Consider the limit augmentation $\mu^{\prime}=[\mathcal{A} ; \phi, \infty]$.

With the notation of the introduction, $\mu^{\prime}$ has finite depth equal to one.

\subsection{Unicity of limit augmentations.}

Lemma 3.6. Let $\mu, \mu^{*}$ be two valuations on $K[x]$ extending $v$. Suppose they admit essential continuous families of augmentations

$$
\mathcal{A}=\left(\rho_{i}\right)_{i \in A}, \quad \rho_{i}=\left[\mu ; \chi_{i}, \beta_{i}\right] ; \quad \mathcal{A}^{*}=\left(\rho_{j}^{*}\right)_{j \in A^{*}}, \quad \rho_{j}^{*}=\left[\mu^{*} ; \chi_{j}^{*}, \beta_{j}^{*}\right] .
$$

of stable degrees $m, m^{*}$, respectively. Consider embeddings $\Gamma_{\mathcal{A}} \hookrightarrow \Lambda, \Gamma_{\mathcal{A}^{*}} \hookrightarrow \Lambda$ into some common ordered group, whose restrictions to $\Gamma$ coincide.

Then, the following conditions are equivalent.

(1) The sets $\mathcal{A}$ and $\mathcal{A}^{*}$ are one cofinal in each other, with respect to the partial ordering $\leq$ of valuations taking values in $\Lambda$.

(2) $m=m^{*}$ and $\rho_{\mathcal{A}}=\rho_{\mathcal{A}^{*}}$.

(3) $m=m^{*}$ and there is a valuation $\nu$ such that $\rho_{i}, \rho_{j}^{*}<\nu$ for all $i \in A, j \in A^{*}$. If these conditions hold, we say that the continuous families $\mathcal{A}$ and $\mathcal{A}^{*}$ are equivalent.

Proof. Suppose that $\mathcal{A}$ and $\mathcal{A}^{*}$ are one cofinal in each other. By the criterion of equation (5), the two families have the same stable polynomials and $\rho_{\mathcal{A}}=\rho_{\mathcal{A}^{*}}$.

Also, there exist $i, k \in A, j \in A^{*}$ such that $\rho_{i}<\rho_{j}^{*}<\rho_{k}$. By Corollary 2.6 (3), the stable degrees coincide: $m=\operatorname{deg}\left(\rho_{i}\right)=\operatorname{deg}\left(\rho_{j}^{*}\right)=m^{*}$. Thus, (1) implies (2). 
Now, suppose $m=m^{*}$ and $\rho_{\mathcal{A}}=\rho_{\mathcal{A}^{*}}$. That is, $\mathcal{A}$ and $\mathcal{A}^{*}$ have the same stable polynomials, and the stable values of these polynomials coincide.

If $\mathcal{A}$ has a stable limit, then $\nu=\rho_{\mathcal{A}}=\rho_{\mathcal{A}^{*}}$ satisfies condition (3).

Otherwise, for any $\phi \in \mathrm{KP}_{\infty}(\mathcal{A})=\mathrm{KP}_{\infty}\left(\mathcal{A}^{*}\right)$ the limit augmentation valuation

$$
\nu=[\mathcal{A} ; \phi, \infty]=\left[\mathcal{A}^{*} ; \phi, \infty\right]
$$

is a common upper bound of $\mathcal{A}$ and $\mathcal{A}^{*}$. Thus, (2) implies (3).

Condition (3), implies that the set $\mathcal{A} \cup \mathcal{A}^{*}$ is totally ordered, by Theorem 2.4 , Now, suppose that $\mathcal{A}$ is not cofinal in $\mathcal{A}^{*}$. There exists an index $j \in A^{*}$ such that $\rho_{j}^{*}>\rho_{i}$ for all $i \in A$. For any $k>j$ in $A^{*}$, we have

$$
\rho_{j}^{*}\left(\chi_{k}^{*}\right)=\beta_{j}^{*}<\beta_{k}^{*}=\rho_{k}^{*}\left(\chi_{k}^{*}\right) .
$$

This implies that $\chi_{k}^{*}$ is not $\mathcal{A}$-estable. Since $\operatorname{deg}\left(\chi_{k}^{*}\right)=m^{*}=m$, this contradicts the fact that $\mathcal{A}$ is essential. Thus, (3) implies (1).

Let us check when different building data $\phi, \gamma$ lead to the same limit augmentation.

Lemma 3.7. Let $\mathcal{A}$ and $\mathcal{A}^{*}$ be essential continuous families of augmentations as in (8)). Take $\phi \in \mathrm{KP}_{\infty}(\mathcal{A}), \phi^{*} \in \mathrm{KP}_{\infty}\left(\mathcal{A}^{*}\right)$. Choose $\gamma, \gamma^{*} \in \Lambda \infty$ such that $\rho_{i}(\phi)<\gamma$ for all $i \in A$, and $\rho_{j}^{*}\left(\phi^{*}\right)<\gamma^{*}$ for all $j \in A^{*}$.

Then, $\mu^{\prime}=[\mathcal{A} ; \phi, \gamma]$ coincides with $\left(\mu^{\prime}\right)^{*}=\left[\mathcal{A}^{*} ; \phi^{*}, \gamma^{*}\right]$ if and only if

$$
\rho_{\mathcal{A}}=\rho_{\mathcal{A}^{*}}, \quad \operatorname{deg}(\phi)=\operatorname{deg}\left(\phi^{*}\right) \quad \text { and } \quad \rho_{\mathcal{A}}\left(\phi^{*}-\phi\right) \geq \gamma=\gamma^{*} .
$$

Proof. If the conditions of (99) hold, we deduce $\mu^{\prime}=\left(\mu^{\prime}\right)^{*}$ by a completely analogous argument to that used in the proof of Lemma 2.8.

Conversely, suppose $\mu^{\prime}=\left(\mu^{\prime}\right)^{*}$. By Proposition 3.5, $\phi, \phi^{*}$ are key polynomials for $\mu^{\prime}$ of minimal degree. Hence, $\operatorname{deg}(\phi)=\operatorname{deg}\left(\phi^{*}\right)$ and Theorem 1.4 shows that

$$
\gamma=\mu^{\prime}(\phi)=\mu^{\prime}\left(\phi^{*}\right)=\left(\mu^{\prime}\right)^{*}\left(\phi^{*}\right)=\gamma^{*} \text {. }
$$

In particular, $\rho_{\mathcal{A}}\left(\phi_{*}-\phi\right)=\mu^{\prime}\left(\phi_{*}-\phi\right) \geq \gamma$. It remains only to show that $\rho_{\mathcal{A}}=\rho_{\mathcal{A}^{*}}$.

By Lemma 3.6, it suffices to see that $\mathcal{A}, \mathcal{A}^{*}$ are cofinal one into each other. Since $\rho_{i}, \rho_{j}^{*}<\mu^{\prime}$ for all $i \in A, j \in A^{*}$, the set $\mathcal{A} \cup \mathcal{A}^{*}$ is totally ordered by Theorem 2.4.

Suppose that $\rho_{j}^{*}>\rho_{i}$ for all $i \in A$, for some index $j \in A^{*}$. Arguing as in the proof of Lemma 3.6, we deduce that for any $k>j$ in $A^{*}$, the key polynomial $\chi_{k}^{*}$ is not $\mathcal{A}$-stable. Since $m^{*}=\operatorname{deg}\left(\chi_{k}^{*}\right)<\operatorname{deg}\left(\phi^{*}\right)=\operatorname{deg}(\phi)$, this contradicts the minimality of $\operatorname{deg}(\phi)$ among all unstable polynomials.

Lemma 3.8. Let $\mathcal{A}=\left(\rho_{i}\right)_{i \in A}$, with $\rho_{i}=\left[\mu ; \chi_{i}, \beta_{i}\right]$, be a continuous family of augmentations of stable degree $m$, of some valuation $\mu$. Let $\Gamma_{\mathcal{A}} \hookrightarrow \Lambda$ be an embedding of ordered groups.

Since all key polynomials $\chi_{i}$ are $\mu$-equivalent, the value $\beta_{\min }:=\mu\left(\chi_{i}\right)$ is independent of $i \in A$. Let $S$ be the initial segment of $\Lambda_{>\beta_{\min }}$ generated by the set $\left\{\beta_{i} \mid i \in A\right\}$. For all $\beta \in S$, let $\rho_{\beta}=\left[\mu ; \chi_{i}, \beta\right]$, for some $i \in A$ such that $\beta_{i}>\beta$. Then,

(1) If $\mathcal{A}$ has a stable limit, then $\left(\mu, \rho_{\mathcal{A}}\right)_{\Lambda}=\left\{\rho_{\beta} \mid \beta \in S\right\}$, $\operatorname{Deg}\left(\left(\mu, \rho_{\mathcal{A}}\right)_{\Lambda}\right)=\{m\}$.

(2) If $\mathcal{A}$ is essential, let $\mu^{\prime}=[\mathcal{A} ; \phi, \gamma]$ be a limit augmentation for some $\phi \in$ $\mathrm{KP}_{\infty}(\mathcal{A})$ and $\gamma \in \Lambda \infty$ such that $\gamma>T:=\left\{\rho_{j}(\phi) \mid j \in A\right\}$. Then,

$$
\left(\mu, \mu^{\prime}\right)_{\Lambda}=\left\{\rho_{\beta} \mid \beta \in S\right\} \cup\left\{\mu_{\delta} \mid \delta \in \Lambda, T<\delta<\gamma\right\}
$$

where $\mu_{\delta}=[\mathcal{A} ; \phi, \delta]$. In particular, $\operatorname{Deg}\left(\left(\mu, \mu^{\prime}\right)_{\Lambda}\right)=\{m, \operatorname{deg}(\phi)\}$. 
Proof. The valuation $\rho_{\beta}=\left[\mu ; \chi_{i}, \beta\right]$ does not depend on $i$, by Lemma 2.8,

If $\mathcal{A}$ has a stable limit, let us denote $\mu^{\prime}=\rho_{\mathcal{A}}$ as well.

Let $\rho$ be a $\Lambda$-valued valuation such that $\mu<\rho<\mu^{\prime}$. If $\rho \leq \rho_{i}$ for some $i \in A$, then $\rho=\rho_{\beta}$ for some $\beta \in S$, by Lemma 2.7.

Suppose that $\rho>\rho_{i}$ for all $i \in A$. If $\mathcal{A}$ has a stable limit, then $\rho=\rho_{\mathcal{A}}$ by Proposition 3.1, against our assumption that $\rho<\mu^{\prime}$. This proves (1).

If $\mathcal{A}$ has no stable limit, then $\rho$ coincides with $\mu^{\prime}$ on stable polynomials by Corollary 2.5. If $\rho(\phi) \geq \gamma=\mu^{\prime}(\phi)$, then the action of both valuations on $\phi$-expansions shows that $\rho \geq \mu^{\prime}$, against our assumption. Thus, $\rho(\phi)<\gamma=\mu^{\prime}(\phi)$.

This implies that $\phi$ is a key polynomial for $\rho$, by Proposition 2.2. Therefore, for $\delta=\rho(\phi)$, we get $\rho=\mu_{\delta}$, because both valuations coincide on $\phi$-expansions.

\section{MaCLane-Vaquié Chains}

Consider a finite, or countably infinite, chain of mixed augmentations

$$
\mu_{0} \stackrel{\phi_{1}, \gamma_{1}}{\longrightarrow} \mu_{1} \stackrel{\phi_{2}, \gamma_{2}}{\longrightarrow} \cdots \longrightarrow \mu_{n-1} \stackrel{\phi_{n}, \gamma_{n}}{\longrightarrow} \mu_{n} \longrightarrow \cdots
$$

in which every node is an augmentation of the previous node, of one of the following types:

Ordinary augmentation: $\mu_{n+1}=\left[\mu_{n} ; \phi_{n+1}, \gamma_{n+1}\right]$, for some $\phi_{n+1} \in \operatorname{KP}\left(\mu_{n}\right)$.

Limit augmentation: $\mu_{n+1}=\left[\mathcal{A} ; \phi_{n+1}, \gamma_{n+1}\right]$, for some $\phi_{n+1} \in \operatorname{KP}_{\infty}(\mathcal{A})$, where $\mathcal{A}$ is an essential continuous family of augmentations of $\mu_{n}$.

Let us fix $\phi_{0} \in \operatorname{KP}\left(\mu_{0}\right)$ a key polynomial of minimal degree, and let $\gamma_{0}=\mu_{0}\left(\phi_{0}\right)$.

The values $\gamma_{n}$ belong to $\Lambda \infty$ for some ordered group $\Lambda$. For all $n \geq 0$, we shall refer to $\left(\phi_{n}, \gamma_{n}\right)$ as the building pair of $\mu_{n}$.

The following properties of an infinite chain of mixed augmentations follow from Theorem 1.11, Propositions 2.1, 2.2, 3.5 and Corollary 2.5.

- $\gamma_{n}=\mu_{n}\left(\phi_{n}\right)<\gamma_{n+1}$.

- $\phi_{n}$ is a key polynomial for $\mu_{n}$ of minimal degree; thus,

$$
\begin{gathered}
\operatorname{deg}\left(\mu_{n}\right)=\operatorname{deg}\left(\phi_{n}\right) \mid \operatorname{deg}\left(\Phi_{\mu_{n}, \mu_{n+1}}\right) . \\
\text { - } \Phi_{\mu_{n}, \mu_{n+1}}=\left\{\begin{array}{lll}
{\left[\phi_{n+1}\right]_{\mu_{n}},} & \text { if } \mu_{n} \rightarrow \mu_{n+1} \text { ordinary augmentation, } \\
\Phi_{\mu_{n}, \mathcal{A}}=\left[\chi_{i}\right]_{\mu_{n}}, \forall i \in A, & \text { if } \mu_{n} \rightarrow \mu_{n+1} \text { limit augmentation. }
\end{array}\right. \\
\operatorname{deg}\left(\Phi_{\mu_{n}, \mu_{n+1}}\right)=\left\{\begin{array}{lll}
\operatorname{deg}\left(\phi_{n+1}\right), & \text { if } \mu_{n} \rightarrow \mu_{n+1} \text { ordinary augmentation, } \\
\operatorname{stable~degree~of~} \mathcal{A}, & \text { if } \mu_{n} \rightarrow \mu_{n+1} & \text { limit augmentation. }
\end{array}\right.
\end{gathered}
$$

4.1. Definition of MacLane-Vaquié chains. Since we want our chains of mixed augmentations to be as unique as possible, we shall impose a technical condition.

Definition 4.1. A finite, or countably infinite, chain of mixed augmentations as in (10) is a MacLane-Vaquié (abbreviated MLV) chain if every augmentation step satisfies:

- If $\mu_{n} \rightarrow \mu_{n+1}$ is ordinary, then $\operatorname{deg}\left(\mu_{n}\right)<\operatorname{deg}\left(\Phi_{\mu_{n}, \mu_{n+1}}\right)$.

- If $\mu_{n} \rightarrow \mu_{n+1}$ is limit, then $\operatorname{deg}\left(\mu_{n}\right)=\operatorname{deg}\left(\Phi_{\mu_{n}, \mu_{n+1}}\right)$ and $\phi_{n} \notin \Phi_{\mu_{n}, \mu_{n+1}}$.

A MacLane-Vaquié chain is complete if the valuation $\mu_{0}$ has depth zero. 
Let us point out some specific features of complete infinite MLV-chains. Denote

$$
m_{n}=\operatorname{deg}\left(\mu_{n}\right)=\operatorname{deg}\left(\phi_{n}\right) \quad \text { for all } \quad n \geq 0 \text {. }
$$

- $m_{n}<m_{n+1}$.

If $\mu_{n} \rightarrow \mu_{n+1}$ is an ordinary augmentation, then $m_{n}<\operatorname{deg}\left(\phi_{n+1}\right)$ by the MLV condition.

If $\mu_{n} \rightarrow \mu_{n+1}$ is a limit augmentation, then $m_{n}$ is the stable degree of the essential continuous family $\mathcal{A}$ of augmentations of $\mu_{n}$. Since $\phi_{n+1} \in \mathrm{KP}_{\infty}(\mathcal{A})$, we have $\operatorname{deg}\left(\phi_{n+1}\right)>m_{n}$.

- $\Phi_{\mu_{n}, \mu_{n+1}}$ is a proper class of key polynomials for $\mu$.

Indeed, $\phi_{n}$ is a key polynomial for $\mu_{n}$ of minimal degree, and $\phi_{n} \notin \Phi_{\mu_{n}, \mu_{n+1}}$.

- $\mu_{n}$ is residually transcendental and $\gamma_{n} \in \Gamma_{\mathbb{Q}}$.

Since $\mu_{n}$ admits proper classes of key polynomials, it is commensurable over $v$ by Theorem 1.9.

However, in a finite MLV-chain of length $r$, the value $\gamma_{r}$ may not belong to $\Gamma_{\mathbb{Q}}$, or be equal to $\infty$. Thus, $\mu_{r}$ may be incommensurable, or have non-trivial support.

- Every infinite MacLane-Vaquié chain has a stable limit.

Indeed, any $f \in K[x]$ has $\operatorname{deg}(f)<m_{n}$ for some $n$. Hence, $\mu_{n}(f)=\mu_{n+1}(f)$ by Proposition 2.2 , since $m_{n} \leq \operatorname{deg}\left(\Phi_{\mu_{n}, \mu_{n+1}}\right)$ for both types of the augmentation $\mu_{n} \rightarrow \mu_{n+1}$.

The criterion of equation (5) shows that $f$ is stable.

- By Propositions 2.1 and 3.5, we get a chain of value groups:

(11) $\quad \Gamma_{\mu_{-1}}:=\Gamma \subset \Gamma_{\mu_{0}} \subset \cdots \subset \Gamma_{\mu_{n}} \subset \cdots \quad$ with $\quad \Gamma_{\mu_{n}}=\left\langle\Gamma_{\mu_{n-1}}, \gamma_{n}\right\rangle, \forall n \geq 0$.

- $\Gamma_{\mu_{n}, m_{n}}=\Gamma_{\mu_{n-1}}$. In particular, $e_{n}:=e_{\text {rel }}\left(\mu_{n}\right)=\left(\Gamma_{\mu_{n}}: \Gamma_{\mu_{n-1}}\right)$.

Indeed, take any $a \in K[x]$ such that $\operatorname{deg}(a)<m_{n}$.

If $\mu_{n-1} \rightarrow \mu_{n}$ is an ordinary augmentation, then $\mu_{n-1}(a)=\mu_{n}(a)$, because $m_{n}=$ $\operatorname{deg}\left(\Phi_{\mu_{n-1}, \mu_{n}}\right)$. Thus, $\Gamma_{\mu_{n}, m_{n}} \subset \Gamma_{\mu_{n-1}}$. On the other hand, $\phi_{n}$ is a proper key polynomial for $\mu_{n-1}$ and Lemma 1.7 shows that $\Gamma_{\mu_{n-1}}=\Gamma_{\mu_{n-1}, m_{n}} \subset \Gamma_{\mu_{n}, m_{n}}$.

If $\mu_{n}=\left[\mathcal{A} ; \phi_{n}, \gamma_{n}\right]$ is a limit augmentation, then $a$ is $\mathcal{A}$-stable, so that $\mu_{n}(a)=$ $\rho_{\mathcal{A}}(a)$. Thus, $\Gamma_{\mu_{n}, m_{n}} \subset \Gamma_{\mathcal{A}}=\Gamma_{\mu_{n-1}}$, the last equality by Lemma 3.4.

Also, the proper class $\Phi_{\mu_{n-1}, \mu_{n}}$ has degree $m_{n-1}<m_{n}$. By Lemma 1.7,

$$
\Gamma_{\mu_{n-1}}=\Gamma_{\mu_{n-1}, m_{n-1}}=\Gamma_{\mu_{n}, m_{n-1}} \subset \Gamma_{\mu_{n}, m_{n}} .
$$

\subsection{The main theorem of MacLane-Vaquié.}

Lemma 4.2. Let $\left(\mu_{n}\right)_{0 \leq n \leq r}$ be a complete finite $M L V$-chain. Let $\mu_{r} \stackrel{\phi, \gamma}{\longrightarrow} \mu$ be an ordinary, or limit augmentation. Then, $\mu$ is the last valuation of a complete finite $M L V$-chain, keeping $(\phi, \gamma)$ as the building pair of $\mu$ in the last augmentation step.

Proof. If the augmentation step $\mu_{r} \rightarrow \mu$ satisfies the MLV condition of Definition 4.1, the statement is obvious. Let us suppose that this is not the case.

Case 1. The augmentation $\mu=\left[\mu_{r} ; \phi, \gamma\right]$ is ordinary and $\operatorname{deg}\left(\mu_{r}\right)=\operatorname{deg}(\phi)$. 
If $r=0$, then $\phi=x-a$ for some $a \in K$ and $\mu$ is a depth zero valuation $\mu=\omega_{a, \gamma}$. Thus, $\mu$ forms a complete MLV-chain of length zero whose building pair is $(\phi, \gamma)$.

If $r>0$ and $\mu_{r}=\left[\mu_{r-1} ; \phi_{r}, \gamma_{r}\right]$ is an ordinary augmentation, then

$$
\operatorname{deg}\left(\mu_{r-1}\right)<\operatorname{deg}\left(\phi_{r}\right)=\operatorname{deg}\left(\mu_{r}\right)=\operatorname{deg}(\phi) .
$$

By Corollary 2.5,

$$
\phi \in \Phi_{\mu_{r}, \mu} \subset \Phi_{\mu_{r-1}, \mu}=\Phi_{\mu_{r-1}, \mu_{r}}=\left[\phi_{r}\right]_{\mu_{r-1}} .
$$

Hence, $\phi$ is a key polynomial for $\mu_{r-1}$ and the ordinary augmentation $\left[\mu_{r-1} ; \phi, \gamma\right]$ satisfies the MLV condition. Also, $\left[\mu_{r-1} ; \phi, \gamma\right]=\mu$ because both valuations coincide on $\phi$-expansions.

If $r>0$ and $\mu_{r}=\left[\mathcal{A} ; \phi_{r}, \gamma_{r}\right]$ is a limit augmentation, then

$$
\operatorname{deg}\left(\mu_{r-1}\right)=\operatorname{deg}\left(\Phi_{\mu_{r-1}, \mu_{r}}\right)=\text { stable degree of } \mathcal{A}<\operatorname{deg}\left(\phi_{r}\right)=\operatorname{deg}(\phi) .
$$

Since $\phi$ is a key polynomial for $\mu_{r}, \operatorname{in}_{\mu_{r}} \phi$ is not a unit. By Proposition 3.5 (2), $\phi$ is unstable of minimal degree for the family $\mathcal{A}$; thus, $\phi \in \mathrm{KP}_{\infty}(\mathcal{A})$. As before, $\mu=[\mathcal{A} ; \phi, \gamma]$ and this limit augmentation satisfies the MLV condition, because the essential continuous family $\mathcal{A}$ of augmentations of $\mu_{r-1}$ remains unchanged.

Case 2. The augmentation $\mu=[\mathcal{A} ; \phi, \gamma]$ is a limit augmentation with respect to some essential continuous family $\mathcal{A}=\left(\rho_{i}\right)_{i \in A}$ of augmentations of $\mu_{r}$. Let $\rho_{i}=\left[\mu_{r} ; \chi_{i}, \beta_{i}\right]$.

Take any $i \in A$. We may decompose the augmentation step $\mu_{r} \rightarrow \mu$ into two pieces:

$$
\mu_{r} \stackrel{\chi_{i}, \beta_{i}}{\longrightarrow} \rho_{i} \stackrel{\phi, \gamma}{\longrightarrow} \mu,
$$

where $\mu_{r} \rightarrow \rho_{i}$ is an ordinary augmentation, and $\mu=\left[\mathcal{A}_{>i} ; \phi, \gamma\right]$ is a limit augmentation with respect to the essential continuous family $\mathcal{A}_{>i}$ of augmentations of $\rho_{i}$.

By the previous case, $\rho_{i}$ is the last valuation of a complete finite MLV-chain keeping $\left(\chi_{i}, \beta_{i}\right)$ as the building pair of $\rho_{i}$. Also, the limit augmentation $\rho_{i} \rightarrow \mu$ satisfies the MLV condition. Indeed, the class

$$
\Phi_{\rho_{i}, \mu}=\Phi_{\rho_{i}, \mathcal{A}_{>i}}=\left[\chi_{j}\right]_{\rho_{i}} \text { for all } j>i
$$

has degree $\operatorname{deg}\left(\chi_{j}\right)=\operatorname{deg}\left(\rho_{i}\right)$, and $\chi_{i} \notin \Phi_{\rho_{i}, \mu}$ because $\chi_{i} \chi_{\rho_{i}} \chi_{j}$ for all $j>i$, by condition (3) of Definition 3.2 .

Theorem 4.3. Every valuation $\nu$ on $K[x]$ falls in one of the following cases.

(a) It is the last valuation of a complete finite MacLane-Vaquié chain.

$$
\mu_{0} \stackrel{\phi_{1}, \gamma_{1}}{\longrightarrow} \mu_{1} \stackrel{\phi_{2}, \gamma_{2}}{\longrightarrow} \cdots \longrightarrow \mu_{r-1} \stackrel{\phi_{r}, \gamma_{r}}{\longrightarrow} \mu_{r}=\nu
$$

(b) It is the stable limit of a continuous family $\mathcal{A}=\left(\rho_{i}\right)_{i \in A}$ of augmentations of some valuation $\mu_{r}$ falling in case (a):

$$
\mu_{0} \stackrel{\phi_{1}, \gamma_{1}}{\longrightarrow} \mu_{1} \stackrel{\phi_{2}, \gamma_{2}}{\longrightarrow} \cdots \longrightarrow \mu_{r-1} \stackrel{\phi_{r}, \gamma_{r}}{\longrightarrow} \mu_{r} \stackrel{\left(\rho_{i}\right)_{i \in A}}{\longrightarrow} \rho_{\mathcal{A}}=\nu
$$

such that the class $\Phi_{\mu_{r}, \nu}$ has degree $\operatorname{deg}\left(\mu_{r}\right)$ and $\phi_{r} \notin \Phi_{\mu_{r}, \nu}$.

(c) It is the stable limit of a complete infinite MacLane-Vaquié chain.

$$
\mu_{0} \stackrel{\phi_{1}, \gamma_{1}}{\longrightarrow} \mu_{1} \stackrel{\phi_{2}, \gamma_{2}}{\longrightarrow} \cdots \longrightarrow \mu_{n-1} \stackrel{\phi_{n}, \gamma_{n}}{\longrightarrow} \mu_{n} \longrightarrow \cdots
$$

We say that $\mu$ has finite depth $r$, quasi-finite depth $r$, or infinite depth, respectively. 
Proof. If $\nu$ is a valuation of depth zero, then it obviously falls in case (a).

Otherwise, let $\gamma_{0}=\nu(x)<\infty$. The depth zero valuation $\mu_{0}=\omega_{0, \gamma_{0}}$ forms a complete finite MLV-chain of length zero, whose last valuation satisfies $\mu_{0}<\nu$.

In order to prove the theorem, it suffices to prove the following Claim.

Claim. For any complete finite MLV-chain of length $r \geq 0$, whose last valuation $\mu_{r}$ satisfies $\mu_{r}<\nu$, at least one of the two following conditions hold:

(1) The valuation $\nu$ is the stable limit of some continuous family of augmentations of $\mu_{r}$.

(2) We may apply to $\mu_{r}$ one, or two, augmentation steps and build up a valuation $\mu_{r}<\mu \leq \nu$ such that either $\mu=\nu$, or $\operatorname{deg}\left(\mu_{r}\right)<\operatorname{deg}(\mu)$.

Indeed, suppose that $\nu=\rho_{\mathcal{A}}$ is the stable limit of some continuous family $\mathcal{A}=$ $\left(\rho_{i}\right)_{i \in A}$ of augmentations of $\mu_{r}$. For any choice of $i \in A$, we may split the step $\mu_{r} \rightarrow \nu$ into two pieces

$$
\mu_{r} \stackrel{\chi_{i}, \beta_{i}}{\longrightarrow} \rho_{i} \stackrel{\left(\rho_{j}\right)_{j>i}}{\longrightarrow} \rho_{\mathcal{A}>i}=\nu
$$

where $\mu_{r} \rightarrow \rho_{i}$ is an ordinary augmentation, and $\nu$ is the stable limit of the continuous family $\mathcal{A}_{>i}$ of augmentations of $\rho_{i}$. Arguing as in the proof of Case 2 in Lemma 4.2, we see that $\nu$ falls in case (b) of the theorem.

On the other hand, if (2) holds, then the valuation $\mu$ is the last valuation of a complete finite MLV-chain by Lemma 4.2 .

Therefore, the iteration of this procedure shows that either $\nu$ falls in cases (a) or (b) of the theorem, or there exists a complete infinite MLV-chain $\left(\mu_{n}\right)_{n>0}$ satisfying $\mu_{n}<\nu$ for all $n$. The stable limit of this MLV-chain is $\nu$ by Proposition 3.1. Thus, $\nu$ falls in case (c).

Let us prove the Claim. Let $m=\operatorname{deg}\left(\Phi_{\mu_{r}, \nu}\right)$. If $m>\operatorname{deg}\left(\mu_{r}\right)$, then for any $\phi \in \Phi_{\mu_{r}, \nu}$ the augmented valuation $\mu=\left[\mu_{r} ; \phi, \nu(\phi)\right]$ satisfies the Claim.

Suppose that $m=\operatorname{deg}\left(\mu_{r}\right)$. We distinguish two cases according to possible upper bounds of the totally ordered set

$$
A=\nu\left(\Phi_{\mu_{r}, \nu}\right) \subset \Gamma_{\nu} \infty
$$

\section{The set $A$ contains a maximal element.}

Let $\gamma=\operatorname{Max}(A)=\nu(f)$, for some $f \in \Phi_{\mu_{r}, \nu}$. By Proposition 2.2, $f \in \operatorname{KP}\left(\mu_{r}\right)$ and the augmented valuation $\eta=\left[\mu_{r} ; f, \gamma\right]$ satisfies $\eta \leq \nu$. If $\eta=\nu$, then $\mu=\eta$ satisfies the Claim.

Suppose $\eta<\nu$, and let $\Phi_{\eta, \nu}=[\chi]_{\eta}$. By Proposition $2.1, \operatorname{deg}(\eta)=\operatorname{deg}(f)=m$. Since $\chi$ is a key polynomial for $\eta$, we have $\operatorname{deg}(\chi) \geq m$.

If $\operatorname{deg}(\chi)=m$, then $\eta(f)=\eta(\chi)$ by Theorem 1.4, This contradicts the maximality of $\gamma$ :

$$
\gamma=\eta(f)=\eta(\chi)<\nu(\chi), \quad \nu(\chi) \in A .
$$

Thus, $\operatorname{deg}(\chi)>m$ and the augmentation $\mu=[\eta ; \chi, \nu(\chi)]$ satisfies the Claim.

The set $A$ does not contain a maximal element.

By Proposition 3.3, there is a continuous family $\mathcal{A}=\left(\rho_{i}\right)_{i \in A}$ of augmentations of $\mu_{r}$ of stable degree $m$, parameterized by the set $A$, such that $\rho_{i}<\nu$ for all $i \in A$, and all polynomials of degree $m$ are stable. 
If $\mathcal{A}$ has a stable limit $\rho_{\mathcal{A}}$, then $\rho_{\mathcal{A}}=\nu$ by Proposition 3.1. Thus, the Claim is satisfied.

If $\mathcal{A}$ has no stable limit, then it is essential and we may consider the limit augmentation $\mu=[\mathcal{A} ; \phi, \nu(\phi)]$, for some $\phi \in \mathrm{KP}_{\infty}(\mathcal{A})$. Clearly, $\mu \leq \nu$ by the comparison of both valuations on $\phi$-expansions.

If $\mu<\nu$, then Proposition 3.5 shows that $\operatorname{deg}(\mu)=\operatorname{deg}(\phi)>m=\operatorname{deg}\left(\mu_{r}\right)$. Thus, the valuation $\mu$ satisfies the Claim.

Corollary 4.4. With the above notation, let $m_{n}=\operatorname{deg}\left(\mu_{n}\right)$ for all $n \geq 0$. Then, $\operatorname{Deg}\left((-\infty, \nu)_{\Lambda}\right)= \begin{cases}\left\{1=m_{0}, m_{1}, \ldots, m_{r}\right\}, & \text { if } \nu \text { has finite or quasi-finite depth } r, \\ \left\{m_{n} \mid n \geq 0\right\}, & \text { if } \nu \text { has infinite depth. }\end{cases}$

Proof. Since $(-\infty, \nu)_{\Lambda}$ is totally ordered, we have

$$
(-\infty, \nu)_{\Lambda}=\left(-\infty, \mu_{0}\right)_{\Lambda} \cup\left[\mu_{0}, \mu_{1}\right)_{\Lambda} \cup \cdots \cup\left[\mu_{n-1}, \mu_{n}\right)_{\Lambda} \cup \cdots
$$

In equation (4), we find a description of $\left(-\infty, \mu_{0}\right)_{\Lambda}$. Also, Lemmas 2.7 and 3.8 describe all subintervals $\left[\mu_{n}, \mu_{n+1}\right)_{\Lambda}$, and the subinterval $\left[\mu_{r}, \nu\right)_{\Lambda}$ if $\nu$ has quasi-finite depth. The result follows easily from these descriptions.

Therefore, the length $r$ of a MLV-chain of $\nu$, and the degrees $m_{n}=\operatorname{deg}\left(\mu_{n}\right)$ of the nodes of the chain, are intrinsic data of $\nu$.

In particular, the three situations of Theorem 4.3 are mutually exclusive.

Lemma 4.5. Let $\nu$ be a valuation on $K[x]$, with support $\mathfrak{p} \in \operatorname{Spec}(K[x])$.

It falls in case (a) of Theorem 4.3 if and only if $\mathrm{KP}(\nu) \neq \emptyset$, or $\mathfrak{p} \neq 0$.

It falls in case (b) (or case (c)) of Theorem 4.3 if and only if $\operatorname{KP}(\nu)=\emptyset, \mathfrak{p}=0$ and $\nu$ has finite (or infinite) degree, respectively.

Indeed, suppose that $\nu$ admits a complete finite MLV-chain of length $r$. If $\gamma_{r}=\infty$, then $\mathfrak{p}=\phi_{r} K[x]$. If $\gamma_{r}<\infty$, then $\operatorname{KP}(\nu) \neq \emptyset$ because $\phi_{r}$ is a key polynomial for $\nu$.

If $\nu$ is the stable limit of a totally ordered family of valuations, it has $\mathfrak{p}=0$ and $\mathrm{KP}(\mu)=\emptyset$ by Proposition 3.1.

If $\nu$ falls in case (c), then clearly $\operatorname{deg}(\nu)=\infty$. Finally, if $\nu$ falls in case (b), the computation of $(\mu, \nu)_{\Gamma_{\nu}}$ in Lemma 3.8 shows that $\operatorname{deg}(\nu)=\operatorname{deg}\left(\mu_{r}\right)$.

We end this section with another immediate consequence of Theorem 4.3.

Let $\Gamma \hookrightarrow \Lambda$ be an embedding of ordered groups and let $\Lambda^{\text {com }} \subset \Lambda$ be the subgroup of all elements whose class in $\Lambda / \Gamma$ is a torsion element.

We say that $\Lambda$ is a small extension of $\Gamma$ if $\Lambda / \Lambda^{\text {com }}$ is a cyclic group.

Corollary 4.6. Let $\nu$ be a valuation on $K[x]$ extending the valuation $v$ on $K$. Then, $\Gamma_{\nu}$ is a small extension of $\Gamma$.

Proof. If $\nu$ falls in cases (b) or (c) of Theorem 4.3, the extension $\Gamma \subset \Gamma_{\mu}$ is commensurable by Proposition 3.1 and Theorem 1.8. Thus, it is a small extension.

In case (a), $\Gamma_{\nu}$ is generated by $\Gamma$ and a finite family $\gamma_{0}, \ldots, \gamma_{r} \in \Gamma_{\nu}$, from which $\gamma_{0}, \ldots, \gamma_{r-1}$ are commensurable over $\Gamma$ (cf. section 4.1).

Thus, $\Gamma_{\nu}$ is obtained as a commensurable extension $\left\langle\Gamma, \gamma_{0}, \ldots, \gamma_{r-1}\right\rangle_{\mathbb{Z}}$ of $\Gamma$, followed by an extension with cyclic quotient. 
4.3. Unicity of MacLane-Vaquié chains. Let $\nu$ be a valuation on $K[x]$. For any positive integer $m$, consider the set:

$$
V_{m}=V_{m}(\nu)=\{\nu(f) \mid f \in K[x] \text { monic, } \operatorname{deg}(f)=m\} \subset \Gamma_{\nu} \infty .
$$

Consider two complete infinite MacLane-Vaquié chains

$$
\begin{aligned}
& \mu_{0} \stackrel{\phi_{1}, \gamma_{1}}{\longrightarrow} \mu_{1} \stackrel{\phi_{2}, \gamma_{2}}{\longrightarrow} \cdots \longrightarrow \mu_{n-1} \stackrel{\phi_{n}, \gamma_{n}}{\longrightarrow} \mu_{n} \longrightarrow \cdots \\
& \mu_{0}^{*} \stackrel{\phi_{1}^{*}, \gamma_{1}^{*}}{\longrightarrow} \mu_{1}^{*} \stackrel{\phi_{2}^{*}, \gamma_{2}^{*}}{\longrightarrow} \cdots \longrightarrow \mu_{n-1}^{*} \stackrel{\phi_{n}^{*}, \gamma_{n}^{*}}{\longrightarrow} \mu_{n}^{*} \longrightarrow \cdots
\end{aligned}
$$

having the same stable limit $\nu$.

By Corollary 4.4, $m_{n}=\operatorname{deg}\left(\mu_{n}\right)=\operatorname{deg}\left(\mu_{n}^{*}\right)$, for all $n \geq 0$.

For any limit augmentation $\mu_{n} \rightarrow \mu_{n+1}$ denote by $\mu_{n, \infty}=\rho_{\mathcal{A}}$ the stability function of the underlying essential continuous family $\mathcal{A}$ of augmentations of $\mu_{n}$. Consider an analogous notation $\mu_{n, \infty}^{*}$ for the second MLV-chain.

Theorem 4.7. For all $n \geq 0, \Gamma_{\mu_{n}}=\Gamma_{\mu_{n}^{*}}$ and the following conditions hold.

(1) If the set $V_{m_{n}}$ defined in (12) contains a maximal element, then

$$
\mu_{n}=\mu_{n}^{*}, \quad \gamma_{n}=\gamma_{n}^{*}=\max \left(V_{m_{n}}\right),
$$

and the augmentation steps $\mu_{n} \rightarrow \mu_{n+1}, \mu_{n} \rightarrow \mu_{n+1}^{*}$ are both ordinary.

(2) If $V_{m_{n}}$ does not contain a maximal element, then $\mu_{n} \rightarrow \mu_{n+1}, \mu_{n}^{*} \rightarrow \mu_{n+1}^{*}$ are limit augmentation steps whose essential continuous families of augmentations are equivalent. In particular, $\mu_{n, \infty}=\mu_{n, \infty}^{*}$.

Proof. Suppose that $V_{m_{n}}$ contains a maximal element.

Take $f \in K[x]$ monic of degree $m_{n}$ such that $\nu(f)=\max \left(V_{m_{n}}\right)$.

Suppose that $\mu_{n} \rightarrow \mu_{n+1}$ is a limit augmentation, and let $\mathcal{A}=\left(\rho_{i}\right)_{i \in A}$, with $\rho_{i}=$ $\left[\mu_{n} ; \chi_{i}, \beta_{i}\right]$, be the corresponding essential continuous family of augmentations of $\mu_{n}$.

By the MLV condition, the stable degree of $\mathcal{A}$ is $m_{n}$; thus, all polynomials in $K[x]$ of degree $m_{n}$ are stable. By Proposition 3.5, there exists $i \in A$ such that $\rho_{i}(f)=\nu(f)$.

On the other hand, since $\chi_{i}$ is a key polynomial of degree $m_{n}$ for $\rho_{i}$, Theorem 1.4 shows that $\rho_{i}(f) \leq \rho_{i}\left(\chi_{i}\right)$, and this contradicts the maximality of $\nu(f)$ :

$$
\nu(f)=\rho_{i}(f) \leq \rho_{i}\left(\chi_{i}\right)=\beta_{i}<\beta_{j}=\nu\left(\chi_{j}\right) \in V_{m_{n}} .
$$

Therefore, the augmentation step $\mu_{n} \rightarrow \mu_{n+1}$ must be ordinary. By the MLV condition,

$$
\operatorname{deg}(f)=m_{n}<m_{n+1}=\operatorname{deg}\left(\Phi_{\mu_{n}, \mu_{n+1}}\right)=\operatorname{deg}\left(\Phi_{\mu_{n}, \nu}\right) .
$$

Hence, $\mu_{n}(f)=\nu(f)$. Since $\phi_{n} \in \mathrm{KP}\left(\mu_{n}\right)$ has degree $m_{n}$ too, Theorem 1.4 shows that

$$
\nu(f)=\mu_{n}(f) \leq \mu_{n}\left(\phi_{n}\right)=\gamma_{n}=\nu\left(\phi_{n}\right) \in V_{m_{n}} .
$$

Since $\nu(f)$ is maximal, we must have $\gamma_{n}=\nu(f)=\max \left(V_{m_{n}}\right)$.

This argument is valid for both MLV-chains. Thus, the augmentation steps $\mu_{n} \rightarrow$ $\mu_{n+1}, \mu_{n}^{*} \rightarrow \mu_{n+1}^{*}$ are both ordinary and $\gamma_{n}=\gamma_{n}^{*}=\max \left(V_{m_{n}}\right)$.

Finally, it is obvious that $\mu_{n} \leq \mu_{n}^{*}$ by comparing the action of both valuations on $\phi_{n}$-expansions. A symmetric argument shows that $\mu_{n}=\mu_{n}^{*}$.

Now, suppose that $V_{m_{n}}$ does not contain a maximal element. 
If the augmentation $\mu_{n} \rightarrow \mu_{n+1}$ is ordinary, equations (13) and (14) hold for any monic polynomial $f$ of degree $m_{n}$. They imply $\max \left(V_{m_{n}}\right)=\gamma_{n}$, contradicting the hypothesis.

Thus, $\mu_{n} \rightarrow \mu_{n+1}$ is a limit augmentation.

Since $m_{n}=\operatorname{deg}\left(\mu_{n}\right)=\operatorname{deg}\left(\mu_{n}^{*}\right)$, this argument applies to the second chain too and $\mu_{n}^{*} \rightarrow \mu_{n+1}^{*}$ is a limit augmentation. Let $\mathcal{A}, \mathcal{A}^{*}$ be the underlying essential continuous families of augmentations of $\mu_{n}$ and $\mu_{n}^{*}$, respectively.

By the MLV condition, the stable degree of $\mathcal{A}$ and $\mathcal{A}^{*}$ is $m_{n}$. Since $\mathcal{A}$ and $\mathcal{A}^{*}$ admit the valuation $\nu$ as a common upper bound, Lemma 3.6 shows that they are equivalent.

Finally, since the classes $\Phi_{\mu_{n}, \mu_{n+1}}$ and $\Phi_{\mu_{n}^{*}, \mu_{n+1}^{*}}$ are proper, Lemma 3.4 shows that $\Gamma_{\mu_{n}}=\Gamma_{\mathcal{A}}=\Gamma_{\mathcal{A}^{*}}=\Gamma_{\mu_{n}^{*}}$

These arguments yield completely analogous unicity results for MacLane-Vaquié chains of valuations of finite, or quasi-finite depth.

Let $\left(\mu_{n}\right)_{0 \leq n}$ be a MLV-chain of $\nu$ of length $r \in \mathbb{N} \infty$. The different behaviour of ordinary and limit augmentations, as far as unicity is concerned, may be explained too by an analysis of the fibers of the order-preserving degree map

$$
\operatorname{deg}:(-\infty, \nu)_{\Lambda} \longrightarrow \mathbb{N} \text {. }
$$

By Corollary 4.4, a non-empty fiber is of the form $\operatorname{deg}^{-1}\left(m_{n}\right)$ for some $n \geq 0$. If this fiber has a maximal element, this element must be a node $\mu_{n}$ of the chain, and $\mu_{n} \rightarrow \mu_{n+1}$ is necessarily an ordinary augmentation. If this fiber has no maximal element, there is no canonical node of degree $m_{n}$ in the chain.

The following graphic of the degree function illustrates the situation.
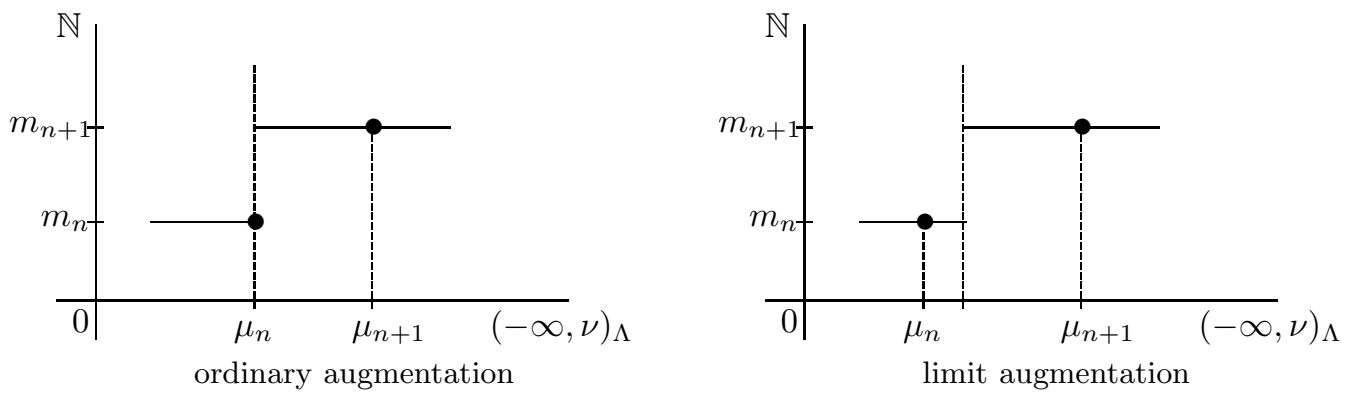

\section{Structure of $\mathcal{G}_{\nu}$ AS a $\mathcal{G}_{v}$-Algebra}

Let $\nu$ be a valuation on $K[x]$. By Theorem 4.3, there is a depth zero valuation $\mu_{0}=\omega_{a_{0}, \gamma_{0}}$ and a finite, or countably infinite, MacLane-Vaquié chain of length $r \in$ $\mathbb{N} \infty$,

$$
\mu_{0} \stackrel{\phi_{1}, \gamma_{1}}{\longrightarrow} \mu_{1} \stackrel{\phi_{2}, \gamma_{2}}{\longrightarrow} \cdots \longrightarrow \mu_{n-1} \stackrel{\phi_{n}, \gamma_{n}}{\longrightarrow} \mu_{n} \longrightarrow \cdots
$$

such that $\nu$ falls in one of the following three cases:

(a) $\nu=\mu_{r}$,

(b) $\nu=\rho_{\mathcal{A}}$ is the stable limit of a continuous family $\mathcal{A}$ of augmentations of $\mu_{r}$, of stable degree $\operatorname{deg}\left(\mu_{r}\right)$. Moreover, $\phi_{r} \notin \Phi_{\mu_{r}, \nu}$.

(c) $\nu=\lim _{n \rightarrow \infty} \mu_{n}$ is the stable limit of the infinite MacLane-Vaquié chain. 
Let $\Gamma_{-1}=\Gamma$ and $\phi_{0}=x-a_{0}$. For all $n \geq 0$, denote $m_{n}=\operatorname{deg}\left(\mu_{n}\right)=\operatorname{deg}\left(\phi_{n}\right)$ and

$$
\Gamma_{n}=\Gamma_{\mu_{n}}, \quad e_{n}=\left(\Gamma_{n}: \Gamma_{n-1}\right), \quad \Delta_{n}=\Delta_{\mu_{n}}, \quad \kappa_{n}=\kappa\left(\mu_{n}\right) .
$$

Since $\mu_{n}$ has finite depth, the following observation follows from Lemma 4.5,

\section{Lemma 5.1.}

(1) $\mathrm{KP}\left(\mu_{n}\right)=\emptyset$ if and only if $n=r$ and $\gamma_{r}=\infty$.

(2) $\mu_{n} / v$ is incommensurable if and only if $n=r$ and $\gamma_{r} \notin \Gamma_{\mathbb{Q}} \infty$.

If $\operatorname{KP}\left(\mu_{n}\right) \neq \emptyset$, we consider the following element in the graded algebra $\mathcal{G}_{\mu_{n}}$ :

$$
q_{n}=\operatorname{in}_{\mu_{n}} \phi_{n} \quad \text { homogeneous prime element of degree } \gamma_{n} .
$$

If $\mu_{n}$ is residually transcendental (that is, $\operatorname{KP}\left(\mu_{n}\right) \neq \emptyset$ and $\mu_{n} / v$ commensurable), then we consider the following elements in $\mathcal{G}_{\mu_{n}}$ :

$$
\begin{array}{ll}
u_{n} & \text { homogeneous unit of degree } e_{n} \gamma_{n} \in \Gamma_{n-1}, \\
\xi_{n}=q_{n}^{e_{n}} u_{n}^{-1} \in \Delta_{n} & \text { Hauptmodul of } \Delta_{n} \text { over } \kappa_{n} .
\end{array}
$$

Let $\rho$ be any valuation such that $\mu_{n}<\rho \leq \nu$. By Corollary 2.6, the canonical homomorphism $\mathcal{G}_{\mu_{n}} \rightarrow \mathcal{G}_{\rho}$ maps the three elements $q_{n}, u_{n}, \xi_{n}$ to homogeneous units in $\mathcal{G}_{\rho}$, which we denote by $x_{n}, u_{n}, z_{n}$, respectively.

In this notation, we omit the reference to the valuation $\rho$. Actually, this will be a general convention on the notation of units of the graded algebras.

Convention. Let $\eta<\rho$ be two valuations on $K[x]$. Given a unit $u \in \mathcal{G}_{\eta}^{*}$, we denote by the same symbol $u \in \mathcal{G}_{\rho}^{*}$ the image of $u$ under the canonical homomorphism $\mathcal{G}_{\eta} \rightarrow \mathcal{G}_{\rho}$

In particular, if $\mu_{n}<\nu$, we get homogeneous units in $\mathcal{G}_{\nu}$ :

$$
\begin{array}{ll}
\text { - } q_{n} \longmapsto x_{n}=\operatorname{in}_{\mu}\left(\phi_{n}\right) & \text { of degree } \gamma_{n} . \\
\text { - } u_{n} \longmapsto u_{n} & \text { of degree } e_{n} \gamma_{n} \in \Gamma_{n-1} . \\
\text { - } \xi_{n} \longmapsto z_{n}=x_{n}^{e_{n}} u_{n}^{-1} \in \kappa(\nu)^{*} & \text { of degree zero. }
\end{array}
$$

Our aim, in this section, is to describe the structure of $\mathcal{G}_{\nu}$ as a $\mathcal{G}_{v}$-algebra, in terms of the discrete data supported by any MacLane-Vaquié chain of $\nu$.

5.1. Computation of $\kappa(\nu)$ and the residue class field $k_{\nu}$. Let $\kappa=\kappa(\nu)$ be the algebraic closure of $k$ in $\Delta=\Delta_{\nu}$.

The canonical homomorphisms $\mathcal{G}_{\mu_{n}} \rightarrow \mathcal{G}_{\mu_{n+1}} \rightarrow \mathcal{G}_{\nu}$ induce a tower of field extensions

$$
k=\kappa_{0} \subset \kappa_{1} \subset \cdots \subset \kappa_{n} \subset \cdots \subset \kappa .
$$

Our first aim is to show that each field extension $\kappa_{n+1} / \kappa_{n}$ is finite.

Lemma 5.2. Suppose that $\mu_{n}<\nu$ and let $\Phi_{\mu_{n}, \nu}=[\phi]_{\mu_{n}}$. Then, the unit $z_{n} \in \mathcal{G}_{\nu}^{*}$ is algebraic over $\kappa_{n}$ and its minimal polynomial over $\kappa_{n}$ is the residual polynomial $R_{\mu_{n}, \phi_{n}, u_{n}}(\phi)$.

In particular, $\left[\kappa_{n}\left(z_{n}\right): \kappa_{n}\right]=\operatorname{deg}\left(\Phi_{\mu_{n}, \nu}\right) / e_{n} m_{n}$. 
Proof. If $\mu_{n} \rightarrow \mu_{n+1}$ is an ordinary augmentation with $\mu_{n+1} \leq \nu$, then we may take $\phi=\phi_{n+1}$. By the MLV condition, $\operatorname{deg}\left(\phi_{n}\right)<\operatorname{deg}(\phi)$, so that $\phi_{n} \nmid_{\mu_{n}} \phi$.

Otherwise, there is a continuous family $\mathcal{A}$ of augmentations of $\mu_{n}$ such that, either $\mathcal{A}$ is essential and $\mu_{n+1}=\left[\mathcal{A} ; \phi_{n+1}, \gamma_{n+1}\right]$ is a limit augmentation with $\mu_{n+1} \leq \nu$, or $n=r$ and $\nu=\rho_{\mathcal{A}}$ is the stable limit of $\mathcal{A}$ as indicated in case (b) of Theorem 4.3.

In both cases, the class $\Phi_{\mu_{n}, \nu}=[\phi]_{\mu_{n}}$ has degree $m_{n}$ and $\phi_{n}$ does not belong to this class. Thus, we have again $\phi_{n} \nmid_{\mu_{n}} \phi$, by Corollary 1.12

In all three cases, $\phi_{n}$ is a key polynomial for $\mu_{n}$ of minimal degree, and $\phi$ is a key polynomial for $\mu_{n}$ such that $\phi_{n} \nmid \mu_{n} \phi$.

Consider the residual polynomial operator attached to $\mu_{n}$ in section 1.2.

$$
R=R_{\mu_{n}, \phi_{n}, u_{n}}: K[x] \rightarrow \kappa_{n}[y] .
$$

By [8, Thm. 5.3], $\operatorname{in}_{\mu_{n}} \phi$ admits the following decomposition in the graded algebra

$$
\operatorname{in}_{\mu_{n}} \phi=\epsilon R(\phi)\left(\xi_{n}\right) \quad \text { for some unit } \epsilon \in \mathcal{G}_{\mu_{n}}^{*} .
$$

By Corollary 2.6, the homomorphism $\mathcal{G}_{\mu_{n}} \rightarrow \mathcal{G}_{\nu}$ vanishes on $\operatorname{in}_{\mu_{n}} \phi$. Thus, it vanishes on $R(\phi)\left(\xi_{n}\right)$ too; that is, $R(\phi)\left(z_{n}\right)=0$. This ends the proof, because $R(\phi) \in \kappa_{n}[y]$ is a monic irreducible polynomial of degree $\operatorname{deg}(\phi) / e_{n} m_{n}$, by Theorem 1.11.

Lemma 5.3. For any augmentation step $\mu_{n} \rightarrow \mu_{n+1} \leq \nu$, we have $\kappa_{n+1}=\kappa_{n}\left(z_{n}\right)$. Moreover, if $\mu_{n} \rightarrow \mu_{n+1}$ is a limit augmentation, then $\kappa_{n+1}=\kappa_{n}\left(z_{n}\right)=\kappa_{n}$.

Proof. Let $\epsilon \in \kappa_{n+1}^{*}=\Delta_{n+1}^{*}$. If $\operatorname{KP}\left(\mu_{n+1}\right) \neq \emptyset$, then Lemma 1.3 shows that a unit of degree zero in the graded algebra may be written as

$$
\epsilon=\operatorname{in}_{\mu_{n+1}} a, \quad a \in K[x], \quad \operatorname{deg}(a)<m_{n+1}, \quad \mu_{n+1}(a)=0 .
$$

If $\operatorname{KP}\left(\mu_{n+1}\right)=\emptyset$, then $\mu_{n+1}=\nu$ and $\gamma_{n+1}=\infty$, by Lemma 5.1. Thus, $\nu$ may be identified with a valuation on the field $K[x] / \phi_{n+1} K[x]$, of degree $m_{n+1}$ over $K$. By Theorem 1.8, $k=\Delta=k_{\nu}$, so that the equality (17) holds trivially too.

Suppose that the augmentation $\mu_{n} \rightarrow \mu_{n+1}$ is ordinary. Since $\phi_{n+1} \nmid_{\mu_{n}} a$, Proposition 2.1 shows that $\mu_{n}(a)=\mu_{n+1}(a)=0$. Hence, in $\operatorname{in}_{n} a$ belongs to $\Delta_{n}$, and $\epsilon=\operatorname{in}_{\mu_{n+1}} a$ belongs to the image of the ring homomorphism $\Delta_{n} \rightarrow \Delta_{n+1}$. By Theorem 1.10, $\Delta_{n}=\kappa_{n}\left[\xi_{n}\right]$, and we deduce that

$$
\epsilon \in \operatorname{Im}\left(\Delta_{n} \rightarrow \Delta_{n+1}\right)=\kappa_{n}\left[z_{n}\right]=\kappa_{n}\left(z_{n}\right) .
$$

Now, suppose that $\mu_{n} \rightarrow \mu_{n+1}$ is a limit augmentation with respect to

$$
\mathcal{A}=\left(\rho_{i}\right)_{i \in A}, \quad \rho_{i}=\left[\mu_{n} ; \chi_{i}, \beta_{i}\right],
$$

an essential continuous family of augmentations of $\mu_{n}$, of stable degree $m_{n}$.

The condition $\operatorname{deg}(a)<m_{n+1}=\operatorname{deg}\left(\phi_{n+1}\right)$ implies that $a$ is $\mathcal{A}$-stable. Hence, there exists $i \in A$ such that $\rho_{i}(a)=\mu_{n+1}(a)=0$. This means that $\epsilon$ is the image of the unit $\operatorname{in}_{\rho_{i}}(a) \in \kappa\left(\rho_{i}\right)$.

On the other hand, if we apply to the augmentation $\mu_{n} \rightarrow \rho_{i}$ the arguments used in the ordinary case, we obtain $\kappa\left(\rho_{i}\right)=\kappa_{n}\left(z_{n}\right)$ as well, where $z_{n} \in \kappa\left(\rho_{i}\right)$ is the image of $\xi_{n}$ under the homomorphism $\mathcal{G}_{\mu_{n}} \rightarrow \mathcal{G}_{\rho_{i}}$.

The relative ramification index of $\rho_{i}$ is equal to one (section 3.1). Since

$$
\Phi_{\mu_{n}, \mu_{n+1}}=\Phi_{\mu_{n}, \rho_{i}}=\left[\chi_{i}\right]_{\mu_{n}},
$$

Lemma 5.2 shows that $z_{n} \in \kappa\left(\rho_{i}\right)$ has degree $m_{n} / m_{n}=1$ over $\kappa_{n}$. 
Therefore, $\kappa\left(\rho_{i}\right)=\kappa_{n}\left(z_{n}\right)=\kappa_{n}$, and $\epsilon$ is the image of some element in $\kappa_{n}^{*}$.

Theorem 5.4. If $\nu=\mu_{r}$ has finite depth, then

$$
\kappa=k\left(z_{0}, \ldots, z_{r-1}\right), \quad k_{\nu}= \begin{cases}\kappa\left(\xi_{r}\right), & \text { if } \gamma_{r} \in \Gamma_{\mathbb{Q}}, \\ \kappa, & \text { if } \gamma_{r} \notin \Gamma_{\mathbb{Q}} .\end{cases}
$$

Suppose that $\nu=\rho_{\mathcal{A}}$ is the stable limit of a continuous family $\mathcal{A}$ of augmentations of $\mu_{r}$, of stable degree $m_{n}$ and such that $\phi_{r} \notin \Phi_{\mu_{r}, \nu}$. Then,

$$
\kappa=k\left(z_{0}, \ldots, z_{r-1}\right)=k_{\nu} .
$$

If $\nu=\lim _{n \rightarrow \infty} \mu_{n}$ is the stable limit of an infinite MacLane-Vaquié chain, then

$$
\kappa=k\left(z_{0}, \ldots, z_{i}, \ldots\right)=k_{\nu}
$$

Proof. If $\nu=\mu_{r}$ has finite depth, then $\kappa=\kappa_{r}=k\left(z_{0}, \ldots, z_{r-1}\right)$ by Lemma 5.3 .

If $\nu$ has quasi-finite or infinite depth, then $\operatorname{KP}(\mu)=\emptyset$ and every non-zero homogeneous element in $\mathcal{G}_{\nu}$ is a unit, by Proposition 3.1. Hence, any $\epsilon \in \kappa^{*}=\Delta^{*}$ may be written as

$$
\epsilon=\operatorname{in}_{\nu} f, \quad f \in K[x], \quad \nu(f)=0 .
$$

Suppose that $\nu$ is the stable limit of a continuous family $\mathcal{A}=\left(\rho_{i}\right)_{i \in A}$ of augmentations of $\mu_{r}$, of stable degree $m_{r}$ such that $\phi_{r} \notin \Phi_{\mu_{r}, \nu}$.

Since $f$ is stable, there exists an index $i \in A$ such that $\rho_{i}(f)=\nu(f)=0$. Thus, $\epsilon$ is the image of the unit $\operatorname{in}_{\rho_{i}} f \in \kappa\left(\rho_{i}\right)$. Now, the arguments in the proof of Lemma 4.5 show that $\kappa\left(\rho_{i}\right)=\kappa_{r}$. Therefore, we have again $\kappa=\kappa_{r}=k\left(z_{0}, \ldots, z_{r-1}\right)$.

Finally, if $\nu=\lim _{n \rightarrow \infty} \mu_{n}$, there exists an index $n \geq 0$ such that $\mu_{n}(f)=\nu(f)=0$. Thus, $\epsilon$ is the image of the unit $\operatorname{in}_{\mu_{n}} f \in \kappa_{n}$. Therefore, in this case we have

$$
\kappa=\bigcup_{n \geq 0} \kappa_{n} .
$$

The statements about $k_{\nu}$ follow from Proposition 3.1 and Theorems $1.8,1.9,1.10$,

Corollary 5.5. Up to natural isomorphisms, the tower (16) of finite field extensions is independent of the MacLane-Vaquié chain underlying $\nu$.

5.2. Structure of $\mathcal{G}_{\nu}$ as a $\mathcal{G}_{v}$-algebra. We keep the notation $\kappa=\kappa(\nu)$ and $\Delta=\Delta_{\nu}$.

The embedding of graded $k$-algebras $\mathcal{G}_{v} \hookrightarrow \mathcal{G}_{\nu}$ induces an embedding of graded $\kappa$-algebras:

$$
\left(\mathcal{G}_{v} \otimes_{k} \kappa\right) \longleftrightarrow \mathcal{G}_{\nu}
$$

In this section, we find an explicit description of $\mathcal{G}_{\nu}$ as a $\left(\mathcal{G}_{v} \otimes_{k} \kappa\right)$-algebra. Together with Theorem 5.4, which computes $\kappa$ in terms of discrete data of the MacLane-Vaquié chain of $\nu$, we obtain an explicit description of the structure of $\mathcal{G}_{\nu}$ as a $\mathcal{G}_{v}$-algebra.

Lemma 5.6. Let $\mu_{n}<\nu$ be a node of the MacLane-Vaquié chain of $\nu$. For any $\alpha \in \Gamma_{n}$, there exists a homogeneous unit $u \in \mathcal{G}_{\mu_{n}}^{*}$ of degree $\alpha$ if and only if $\alpha \in \Gamma_{n-1}$. In this case,

$$
\left(\mathcal{P}_{\alpha}\left(\mu_{n}\right) / \mathcal{P}_{\alpha}^{+}\left(\mu_{n}\right)\right) \cap \mathcal{G}_{\mu_{n}}^{*}=\kappa_{n}^{*} u
$$


Proof. As we saw in section 4.1, $\Gamma_{n-1}=\Gamma_{n, m_{n}}$. Thus, for all $\alpha \in \Gamma_{n-1}$ there is $a \in K[x]$ such that $\operatorname{deg}(a)<m_{n}$ and $\mu_{n}(a)=\alpha$. By Lemma 1.3, $\operatorname{in}_{\mu_{n}} a$ is a unit of degree $\alpha$.

On the other hand, by (11), any $\alpha \in \Gamma_{n} \backslash \Gamma_{n-1}$ can be written as

$$
\alpha=\ell \gamma_{n}+\beta, \quad \ell \in \mathbb{Z}, \quad \ell \neq 0, \quad \beta \in \Gamma_{n-1} .
$$

If $\ell<0$, Lemma 1.2 shows that $\mathcal{P}_{\alpha}=0$. Suppose that $\ell>0$.

By the previous argument, there exists a unit $u \in \mathcal{G}_{\mu_{n}}^{*}$ of degree $\beta$. Then, $u q_{n}^{\ell}$ has degree $\alpha$. Since $q_{n}$ is a prime element in $\mathcal{G}_{\mu_{n}}$, there is no unit in

$$
\mathcal{P}_{\alpha} / \mathcal{P}_{\alpha}^{+}=\left(u q_{n}^{\ell}\right) \Delta_{n}
$$

Finally, if $u \in \mathcal{G}_{\mu_{n}}^{*}$ is a homogeneous unit of degree $\alpha$, we have

$$
\left(\mathcal{P}_{\alpha} / \mathcal{P}_{\alpha}^{+}\right) \cap \mathcal{G}_{\mu_{n}}^{*}=\left(\Delta_{n} u\right) \cap \mathcal{G}_{\mu_{n}}^{*}=\Delta_{n}^{*} u=\kappa_{n}^{*} u .
$$

This ends the proof of the lemma.

Theorem 5.7. (1) If $\nu=\mu_{r}$ has finite depth, then

$$
\mathcal{G}_{\nu}= \begin{cases}\left(\mathcal{G}_{v} \otimes_{k} \kappa\right)\left[x_{0}, \ldots, x_{r-1}\right], & \text { if } \gamma_{r}=\infty, \\ \left(\mathcal{G}_{v} \otimes_{k} \kappa\right)\left[x_{0}, \ldots, x_{r-1}\right]\left[q_{r}\right], & \text { if } \gamma_{r}<\infty .\end{cases}
$$

If $\gamma_{r}<\infty$, then $q_{r}$ is a homogeneous prime element of degree $\gamma_{r}$ which is transcendental over $\left(\mathcal{G}_{v} \otimes_{k} \kappa\right)\left[x_{0}, \ldots, x_{r-1}\right]$.

(2) Suppose that $\nu$ is the stable limit of a continuous family of augmentations of $\mu_{r}$, of stable degree $m_{r}$, such that $\phi_{r} \notin \Phi_{\mu_{r}, \nu}$. Then,

$$
\mathcal{G}_{\nu}=\left(\mathcal{G}_{v} \otimes_{k} \kappa\right)\left[x_{0}, \ldots, x_{r}\right] .
$$

(3) If $\nu=\lim _{n \rightarrow \infty} \mu_{n}$ is the stable limit of an infinite MacLane-Vaquié chain, then

$$
\mathcal{G}_{\nu}=\left(\mathcal{G}_{v} \otimes_{k} \kappa\right)\left[x_{0}, \ldots, x_{n}, \ldots\right] .
$$

In all cases, $x_{n}$ is a homogeneous unit of degree $\gamma_{n}$, algebraic over

$$
\left(\mathcal{G}_{v} \otimes_{k} \kappa\right)\left[x_{0}, \ldots, x_{n-1}\right],
$$

with minimal equation

$$
x_{n}^{e_{n}}=u_{n} z_{n} \in\left(\mathcal{G}_{v} \otimes_{k} \kappa\right)\left[x_{0}, \ldots, x_{n-1}\right], \quad n \geq 0 .
$$

Proof. For $n>0$, since $\Gamma_{n-1}=\left\langle\Gamma, \gamma_{0}, \ldots, \gamma_{n-1}\right\rangle$ and $x_{0}, \ldots, x_{n-1}$ are units of degree $\gamma_{0}, \ldots, \gamma_{n-1}$ respectively, the subalgebra

$$
\left(\mathcal{G}_{v} \otimes_{k} \kappa\right)\left[x_{0}, \ldots, x_{n-1}\right] \subset \mathcal{G}_{\nu}
$$

contains a homogeneous unit $u_{\alpha}$ of degree $\alpha$, for all $\alpha \in \Gamma_{n-1}$. By Lemma 5.6, this algebra contains all homogeneous units of degree $\alpha$, for all $\alpha \in \Gamma_{n-1}$. Thus, it contains $u_{n}$ and $z_{n}$.

If $\nu=\mu_{r}$ has finite depth and $\gamma_{r}=\infty$, we have $\Gamma_{\nu}=\Gamma_{r-1}$ and $\Delta=\kappa$. For all $\alpha \in \Gamma_{\nu}$,

$$
\mathcal{P}_{\alpha}(\nu) / \mathcal{P}_{\alpha}^{+}(\nu)=\Delta u_{\alpha}=\kappa u_{\alpha} \subset\left(\mathcal{G}_{v} \otimes_{k} \kappa\right)\left[x_{0}, \ldots, x_{r-1}\right] .
$$

Hence, $\left(\mathcal{G}_{v} \otimes_{k} \kappa\right)\left[x_{0}, \ldots, x_{r-1}\right]=\mathcal{G}_{\nu}$.

If $\nu=\mu_{r}$ has finite depth and $\gamma_{r}<\infty$, consider the subalgebra

$$
\mathcal{G}=\left(\mathcal{G}_{v} \otimes_{k} \kappa\right)\left[x_{0}, \ldots, x_{r-1}\right]\left[q_{r}\right] \subset \mathcal{G}_{\nu} .
$$


If $\nu / v$ is incommensurable, then $\Delta=\kappa$ by Theorem 1.9. If $\nu / v$ is commensurable, the algebra $\mathcal{G}$ contains $u_{r}$ by the arguments above. Hence, it contains $\xi_{r}=q_{r}^{e_{r}} u_{r}^{-1}$. Therefore, in all cases $\Delta \subset \mathcal{G} \subset \mathcal{G}_{\nu}$.

Now, let $\alpha \in \Gamma_{r}=\Gamma_{\nu}$ such that $\mathcal{P}_{\alpha} / \mathcal{P}_{\alpha}^{+} \neq 0$. If $\operatorname{in}_{\nu} f$ is a non-zero homogeneous element of degree $\alpha$, Lemma 1.2 shows that

$$
\alpha=\nu(f)=\ell \gamma_{r}+\beta, \quad \ell \in \mathbb{Z}_{\geq 0}, \quad \beta \in \Gamma_{r-1} .
$$

If $u$ is an homogeneous unit in $\left(\mathcal{G}_{v} \otimes_{k} \kappa\right)\left[x_{0}, \ldots, x_{r-1}\right]$ of degree $\beta$, the element $\zeta=q_{r}^{\ell} u$ belongs to $\mathcal{G}$ and has degree $\alpha$.

Hence, $\mathcal{P}_{\alpha} / \mathcal{P}_{\alpha}^{+}=\Delta \zeta$ is contained in $\mathcal{G}$. This proves that $\mathcal{G}=\mathcal{G}_{\nu}$.

This proves (1). The other two cases follow from similar arguments. Actually, the proof is easier in both cases, because $\Delta=\kappa$ and all non-zero homogeneous elements are units.

Corollary 5.8. If $\nu$ has finite depth and trivial support, the subalgebra

$$
\mathcal{G}_{0}=\left(\mathcal{G}_{v} \otimes_{k} \kappa\right)\left[x_{0}, \ldots, x_{r-1}\right]
$$

is the algebraic closure of $\mathcal{G}_{v}$ in $\mathcal{G}_{\nu}=\mathcal{G}_{0}\left[q_{r}\right]$, which is purely transcendental over $\mathcal{G}_{0}$.

In all other cases, the extension $\mathcal{G}_{v} \subset \mathcal{G}_{\nu}$ is algebraic.

We may reinterpretate Theorem 5.7 as an intrinsic construction of $\mathcal{G}_{\nu}$, depending only on the data $e_{n}, \gamma_{n}$ for all $n \geq 0$.

For instance, suppose that $\nu$ has finite depth $r$. We consider indeterminates $x_{0}, \ldots, x_{r-1}$ of degrees $\gamma_{0}, \ldots, \gamma_{r-1}$, submitted to the relations (18).

For $n<r-1$, once the subalgebra $\left(\mathcal{G}_{v} \otimes_{k} \kappa\right)\left[x_{0}, \ldots, x_{n-1}\right]$ has been constructed, we may choose an arbitrary homogeneous unit $u_{n}$ of degree $e_{n} \gamma_{n} \in \Gamma_{n-1}$ in this subalgebra, and this facilitates the construction of $\left(\mathcal{G}_{v} \otimes_{k} \kappa\right)\left[x_{0}, \ldots, x_{n}\right]$.

If $\gamma_{r}=\infty$ we get $\mathcal{G}_{\nu}=\left(\mathcal{G}_{v} \otimes_{k} \kappa\right)\left[x_{0}, \ldots, x_{r-1}\right]$.

If $\gamma_{r}<\infty$, we consider an indeterminate $q_{r}$ of degree $\gamma_{r}$ and we take

$$
\mathcal{G}_{\nu}=\left(\mathcal{G}_{v} \otimes_{k} \kappa\right)\left[x_{0}, \ldots, x_{r-1}\right]\left[q_{r}\right] .
$$

\section{Defect of the extension of a VAluation}

6.1. Numerical data attached to a valuation on $K[x]$. Let $\nu$ be a valuation on $K[x]$ with underlying MacLane-Vaquié chain as in (15).

Keeping with all notation introduced in section 5, we may attach to each node $\mu_{n}<\mu_{n+1} \leq \nu$ of the chain the following numerical data.

The relative ramification index and residual degree, defined as the positive integers

$$
e_{n}=\left(\Gamma_{n}: \Gamma_{n-1}\right), \quad f_{n}=\left[\kappa_{n+1}: \kappa_{n}\right]=\operatorname{deg}\left(\Phi_{\mu_{n}, \nu}\right) / e_{n} m_{n},
$$

respectively. Also, the relative gap is defined as the rational number

$$
d_{n}=\frac{m_{n+1}}{\operatorname{deg}\left(\Phi_{\mu_{n}, \nu}\right)}= \begin{cases}1, & \text { if } \mu_{n} \rightarrow \mu_{n+1} \text { is an ordinary augmentation, } \\ m_{n+1} / m_{n}, & \text { if } \mu_{n} \rightarrow \mu_{n+1} \text { is a limit augmentation. }\end{cases}
$$

By Theorem 4.7 and Corollary [5.5, these numbers are intrinsic data of $\nu$. Clearly,

$$
m_{n+1}=e_{n} f_{n} d_{n} m_{n} \text { for all } n \geq 0 .
$$

For all $n \geq 0$ we may consider the valuation

$$
v_{n}:=\left[\mu_{n} ; \phi_{n}, \infty\right],
$$


with support $\mathfrak{p}_{v_{n}}=\phi_{n} K[x]$. This valuation determines an extension of $v$ to the finite field extension $K_{\phi_{n}} / K$, where $K_{\phi_{n}}=K[x] /\left(\phi_{n} K[x]\right)$. We abuse of language and use the same symbol $v_{n}$ for both valuations. That is,

$$
v_{n}: K[x] \rightarrow K_{\phi_{n}} \stackrel{v_{n}}{\longrightarrow} \Gamma_{\mu_{n}} \infty,
$$

It is well known how to compute the value group and residue class field of this valuation in terms of data of the pair $\mu_{n}, \phi_{n}$ [8, Props. 2.12+3.6]:

$$
\Gamma_{v_{n}}=\Gamma_{\mu_{n}, m_{n}}=\Gamma_{n-1}, \quad k_{v_{n}} \simeq \kappa_{n} .
$$

Let us define $d\left(\mu_{n} / v\right):=m_{n} / e\left(v_{n} / v\right) f\left(v_{n} / v\right)$, where $e\left(v_{n} / v\right), f\left(v_{n} / v\right)$ are the ramificaction index and residual degree of the extension $v_{n} / v$, respectively. Then, we have:

$$
e\left(v_{n} / v\right)=e_{0} \cdots e_{n-1}, \quad f\left(v_{n} / v\right)=f_{0} \cdots f_{n-1}, \quad d\left(\mu_{n} / v\right)=d_{0} \cdots d_{n-1} .
$$

In fact, the formulas for $e\left(v_{n} / v\right)$ and $f\left(v_{n} / v\right)$ follow immediately from (20) and the definition of the numbers $e_{n}, f_{n}$. The formula for $d\left(\mu_{n} / v\right)$ follows from (19)).

6.2. Defect of a valuation on a simple finite extension of fields. Let $L / K$ be a finite simple field extension. That is, $L=K(\theta)$, where $\theta$ is the root of some monic irreducible $F \in K[x]$ in some fixed algebraic closure of $K$.

Let $\nu$ be a valuation on $L$ extending $v$. We abuse of language and use the same symbol to denote the valuation on $K[x]$ determined by $\nu$.

Since $\nu$ has non-trivial support $\mathfrak{p}=F K[x]$, Lemma 4.5] shows that $\nu$ has finite depth and admits a finite MacLane-Vaquié chain

$$
\mu_{0} \stackrel{\phi_{1}, \gamma_{1}}{\longrightarrow} \mu_{1} \stackrel{\phi_{2}, \gamma_{2}}{\longrightarrow} \cdots \longrightarrow \mu_{r-1} \stackrel{\phi_{r}, \gamma_{r}}{\longrightarrow} \mu_{r}=\nu
$$

with $\gamma_{r}=\infty$ and $\phi_{r}=F$. By equations (19) and (21), we have

$$
[L: K]=\operatorname{deg}\left(\phi_{r}\right)=e(\nu / v) f(\nu / v) d(\nu / v) .
$$

If $\eta_{1}, \ldots, \eta_{g}$ are the different extensions of $v$ to $L$, the well known inequality

$$
\sum_{i=1}^{g} e\left(\eta_{i} / v\right) f\left(\eta_{i} / v\right) \leq[L: K]
$$

gives a hint about the information contained in the rational number $d(\nu / v)$.

In particular, if $v$ admits a unique extension $\nu$ to $L$, then the equality in (22) shows that $d(\nu / v)$ coincides with the defect of the extension $\nu / v$.

In this case, $d(\nu / v)=p^{a}$ is an integer, where $p=1$ if $k$ has characteristic zero and $p$ is the characteristic of $k$ if it is positive.

Hence, the computation of (21) yields the following result of Vaquié in [12].

Corollary 6.1. If $\nu$ is the only extension of $v$ to $L$, the defect of the extension $\nu / v$ is the product of the relative gaps of any MacLane-Vaquié chain of $\nu$. 


\section{REFERENCES}

[1] V. Alexandru, N. Popescu, A. Zaharescu, All valuations on $K(x)$, J. Math. Kyoto Univ. 30, Number 2 (1990), 281-296.

[2] F.J. Herrera Govantes, M.A. Olalla Acosta, M. Spivakovsky, Valuations in algebraic field extensions, J. Algebra 312 (2007), no. 2, 1033-1074.

[3] F.J. Herrera Govantes, W. Mahboub, M.A. Olalla Acosta, M. Spivakovsky, Key polynomials for simple extensions of valued fields, preprint, arXiv:1406.0657v4 [math.AG], 2018.

[4] F.-V. Kuhlmann, Value groups, residue fields, and bad places of rational function fields, Trans. Amer. Math. Soc. 356 (2004), no. 11, 4559-4660.

[5] S. MacLane, A construction for absolute values in polynomial rings, Trans. Amer. Math. Soc. 40 (1936), pp. 363-395.

[6] S. MacLane, A construction for prime ideals as absolute values of an algebraic field, Duke Math. J. 2 (1936), pp. 492-510.

[7] N. Moraes de Oliveira, E. Nart, Defectless polynomials over henselian fields and inductive valuations, J. Algebra 541 (2020), 270-307.

[8] E. Nart, Key polynomials over valued fields, Publ. Mat. 64 (2020), 3-42.

[9] M. Vaquié, Famille admisse associée à une valuation de $K[x]$, Singularités Franco-Japonaises, Séminaires et Congrés 10, SMF, Paris (2005), Actes du colloque franco-japonais, juillet 2002, édité par Jean-Paul Brasselet et Tatsuo Suwa, 391-428.

[10] M. Vaquié, Extension d'une valuation, Trans. Amer. Math. Soc. 359 (2007), no. 7, 3439-3481.

[11] M. Vaquié, Algèbre graduée associée à une valuation de K[x], Adv. Stud. Pure Math. 46 (2007), 259-271.

[12] M. Vaquié, Famille admissible de valuations et défaut d'une extension, J. Algebra 311 (2007), no. $2,859-876$.

Departament de Matemàtiques, Universitat Autònoma de Barcelona, Edifici C, E-08193 Bellaterra, Barcelona, Catalonia

Email address: nart@mat.uab.cat 